Article

\title{
A Heterogeneous Battlefield Situation Information Sharing Method Based on Content
}

\author{
Cuntao Liu ${ }^{1, \dagger}$, Wendong Zhao ${ }^{2, *}$, Aijing Li ${ }^{2, *}$ and Junsheng Zhang ${ }^{3,+}$ \\ 1 Graduate School, Army Engineering University of PLA, Nanjing 210000, China; liucuntao@aliyun.com \\ Communications Engineering College, Army Engineering University of PLA, Nanjing 210000, China \\ 3 Key Laboratory of Intelligent Information Processing in Chinese Academy of Sciences, Beijing 100864, China; \\ zhangjs@istic.ac.cn \\ * Correspondence: zhaowendong123@aliyun.com (W.Z.); lishan_wh@126.com (A.L.); \\ Tel.: +86-156-0158-9378 (W.Z.); +86-186-0250-0523 (A.L.) \\ + These authors contributed equally to this work.
}

Received: 17 October 2019; Accepted: 19 December 2019; Published: 25 December 2019

\begin{abstract}
Various information systems adopt different information category standards and description methods even for the same battlefield space. This leads to the heterogeneity of information distributed in different systems, which hinders the information sharing among different systems. In this paper, we adopt the idea of schema mapping and design a framework of realizing heterogeneous information sharing based on content. We design a concept-logic tree model to organize the battlefield situation information and realize the mapping from local concept models in different systems to a global unified concept model. By constructing a unified information space, we realize the centralized organization, storage, and management of entity description information. Then, the information broadcasting mechanism and content based information query mechanism are designed to realize information sharing. Theoretical analysis and experiment results verify the effectiveness of the proposed framework.
\end{abstract}

Keywords: content; heterogeneous; information; semantics; sharing

\section{Introduction}

In the battlefield, realizing an effective acquisition, transmission, and use of battlefield situation information is important for the formulation of a combat plan, deployment of combat forces, and execution of combat operations. Different services and arms have developed various combat information systems with respect to their combat missions, core operational capabilities, and equipment technical conditions.

Currently, joint operations have become the dominant mode of warfare, in which multiple combat information systems coexist. The main reasons include: (1) No single combat information system can meet the diversified operational requirements of different combat units with different services and arms. (2) The development of a new type of universal combat system and the generation of corresponding combat effectiveness are a long term process that needs repeated demonstration, evaluation, and practice test. (3) The original information systems are still playing their roles well in the specific fields, but the capability of a single information system is limited. For example, influenced by the limitation of the reconnaissance scope and reconnaissance capability of reconnaissance equipment, a single information system only describes the information of some operational targets in a specific area of the entire battlefield space. The integrated application of existing combat information systems is the main system application method in the co-existence stage of multiple systems. It is urgent to realize the effective information sharing among different systems. 
Various information systems are different in architecture, technical system, and message standard, which lead to the lack of information inter-operability among different information systems. Besides, in different information systems, different terms may be used to describe the same information, or the same terms may be used to describe different information, which lead to the heterogeneity of semantics. The heterogeneity of information leads to the difficulty of information sharing among systems. Therefore, it has become an urgent problem to realize effective heterogeneous information sharing.

Generally, methods to realize heterogeneous information sharing among different combat information systems include: realizing information interaction between systems by direct message format conversion, realizing system interconnection and information processing by gateway technology, and realizing information sharing among systems by information integration [1-3]. Obviously, compared with simply realizing the information interaction among multiple systems, the integration of multi-system information is more conducive to giving full play to the overall value of information. For example, based on information integration, a single information system can obtain more battlefield target description information in a larger scope than its original information acquisition area, so as to provide better information services for users within the system.

With respect to heterogeneous information integration, techniques such as the federated database system (FDBS) [4], data warehouse (DWH) [5], middleware [6], and schema mapping [7] are proposed, where schema mapping is more convenient to realize information aggregation of existing systems, without changing the current hardware interfaces of combat equipment, and possesses better capability of data processing, as well as compatibility, scalability, and reusability. Meanwhile, using a uniform pattern to describe and organize heterogeneous information is helpful for combat units to understand the meaning of information from different systems correctly. It is beneficial for maintaining the consistency of situational information accessed by each commander and combat unit, which is important for realizing distributed decision-making of commanders of different levels and the coordinated action of combat units.

Following the idea of schema mapping, several studies for realizing heterogeneous battlefield situation information sharing have been done. Among them, the work in [8] used the XML schema as the uniform schema and designed battlefield situation information description models to realize information sharing, which mainly focused on the internal information of target elements, while the semantic relationships among them were less considered. With the development and wide application of ontology technology, the works in [9-12] designed an ontology based information description model to realize schema mapping and produced several advances in realizing effective battlefield situation information sharing. The work in [9] focused on the sharing of battlefield target information; the work in [10] focused on the construction approach of a battlespace ontology based on OWL; the work in [11] focused on the method of realizing battlefield situation collaborative plotting based on ontology; the work in [12] focused on the method of realizing battlefield situational information discovery based on ontology. However, the dynamic and mission related property of the semantic relationships among target elements in the battlefield space are less concerned, but this reflects the dynamic evolution characteristic of the battlefield situation, which is important for commanders to master the real-time state of the battlefield and make decisions. In addition, the graph based organization method of represented information, which is usually used by traditional ontology based systems, is not conducive to improving the efficiency of information searching.

In this paper, we propose a framework of realizing heterogeneous battlefield situation information sharing based on a unified battlefield situation information space. Combat units are the core description objects, and we design a general model to describe the information uniformly in an entity oriented way. To reflect the properties of being dynamic and mission related in semantic relationships, as well as to increase the efficiency of information searching, we organize the external information and the internal description information of entities with different strategies. The main contributions are as follows: 
- We propose an entity oriented description model of battlefield situation information to reflect the basic characteristics of target elements in the battlefield space, as well as the consistency of the heterogeneous battlefield situation information.

- We design a concept-logic tree model to organize heterogeneous information in a consistent way, and a semantic link network is adopted to describe and organize the dynamic and mission related semantic relationships among entities.

- We construct a heterogeneous information sharing framework based on a unified battlefield situation information space, which can realize heterogeneous information sharing among systems.

- Theoretical analysis and experiment results show that the sharing of heterogeneous information among different systems can be realized effectively based on the information sharing framework we propose.

\section{Sharing Framework of Heterogeneous Battlefield Situation Information}

In this section, we first analyze the consistency of heterogeneous battlefield situation information in content under the situation of multiple combat systems in the battlefield space. Then, we introduce the entity oriented formal description model of the battlefield situation in detail. Last, we introduce the layered framework of realizing heterogeneous information sharing based on a unified battlefield situation information space. Table 1 lists the basic notations and the corresponding definitions.

Table 1. A list of major notations.

\begin{tabular}{|c|c|}
\hline Notation & Definition \\
\hline$e$ & $\begin{array}{l}\text { Entity. This represents an independently identifiable, tangible geographical target, combat unit, } \\
\text { or intangible concept. }\end{array}$ \\
\hline$a$ & Attribute variable. This represents an attribute of $e$ \\
\hline$r$ & Relationship variable. This represents a kind of relationship among entities. \\
\hline$s$ & $\begin{array}{l}\text { State variable. This represents an observable state of } e \text {, e.g., the state of motion or operation of an } \\
\text { equipment. It is closely related to the internal attribute information of } e \text {. }\end{array}$ \\
\hline E & Non-empty finite set of entities \\
\hline$A$ & Attribute set. This represents a collection of attribute variables. \\
\hline$R$ & Relationship set. This represents a collection of relationship variables. \\
\hline$S$ & State set. This represents a collection of state variables. \\
\hline EC & Entity class. This is an abstraction of entities that possess the same features. \\
\hline$A_{c}^{\prime}$ & $\begin{array}{l}\text { Feature attribute set. This represents the unique set of attributes possessed by } E C \text {, which is } \\
\text { denoted as } E C \leftrightarrow A_{c}^{\prime} \text {; while the corresponding state set related to } A_{c}^{\prime} \text { is denoted as } S_{c}^{\prime} \text {. }\end{array}$ \\
\hline$A_{c}$ & $\begin{array}{l}\text { Attribute set of } E C \text {, which is denoted as } A_{c} \mapsto E C \text {; while the corresponding state set related to } A_{\mathcal{C}} \\
\text { is denoted as } S_{c} \text {. }\end{array}$ \\
\hline$A_{c c}$ & $\begin{array}{l}\text { The "common-attribute set" of } E C \text {. This is a subset of } A_{c} \text { and represents the set of attributes that } \\
\text { each entity belonging to } E C \text { should possess, which is denoted as } E C \rightarrow A_{c c} ; \text { while the corresponding } \\
\text { state set related to } A_{c c} \text { is denoted as } S_{c c} \text {. }\end{array}$ \\
\hline$A_{c o}$ & $\begin{array}{l}\text { The "occasional-attribute set" of } E C \text {. This is a subset of } A_{c} \text { and represents the set of attributes that } \\
\text { not all entities belonging to } E C \text { should possess; while the corresponding state set related to } A_{c o} \text { is } \\
\text { denoted as } S_{c o} \text {. }\end{array}$ \\
\hline$i, j, k, l, g$ & The mark number of specific $e, a, s, E C, A_{c}$, or $S_{c}$, etc. \\
\hline & The number of $e, a, s, E C, A_{c}$, or $S_{c}$, etc. \\
\hline
\end{tabular}

\subsection{Content Consistency Analysis of Heterogeneous Battlefield Situation Information}

To meet the need for joint operations, the research, development, and assembly of combat systems in the military field are increasingly focused on universality and integration. However, the upgrading of information systems cannot be done overnight. To maintain the continuity of combat effectiveness of the army and the smoothness of equipment evolution, the coexistence of multiple information systems usually is the case for current armies. Take the current U.S. military forces as an example: there are various information systems used by their equipment, such as SADL(Situation Awareness Data Link), Link 16, VMF (Variable Message Format), and Link 22. 
As shown in Figure 1, when conducting joint operations, multiple combat units such as fighters, tanks, ships, satellites, or radar equipped with different information systems may exist in the same combat area at the same time; where any combat unit with communication capabilities can be a provider of battlefield situation information, as well as a user of battlefield situation information.

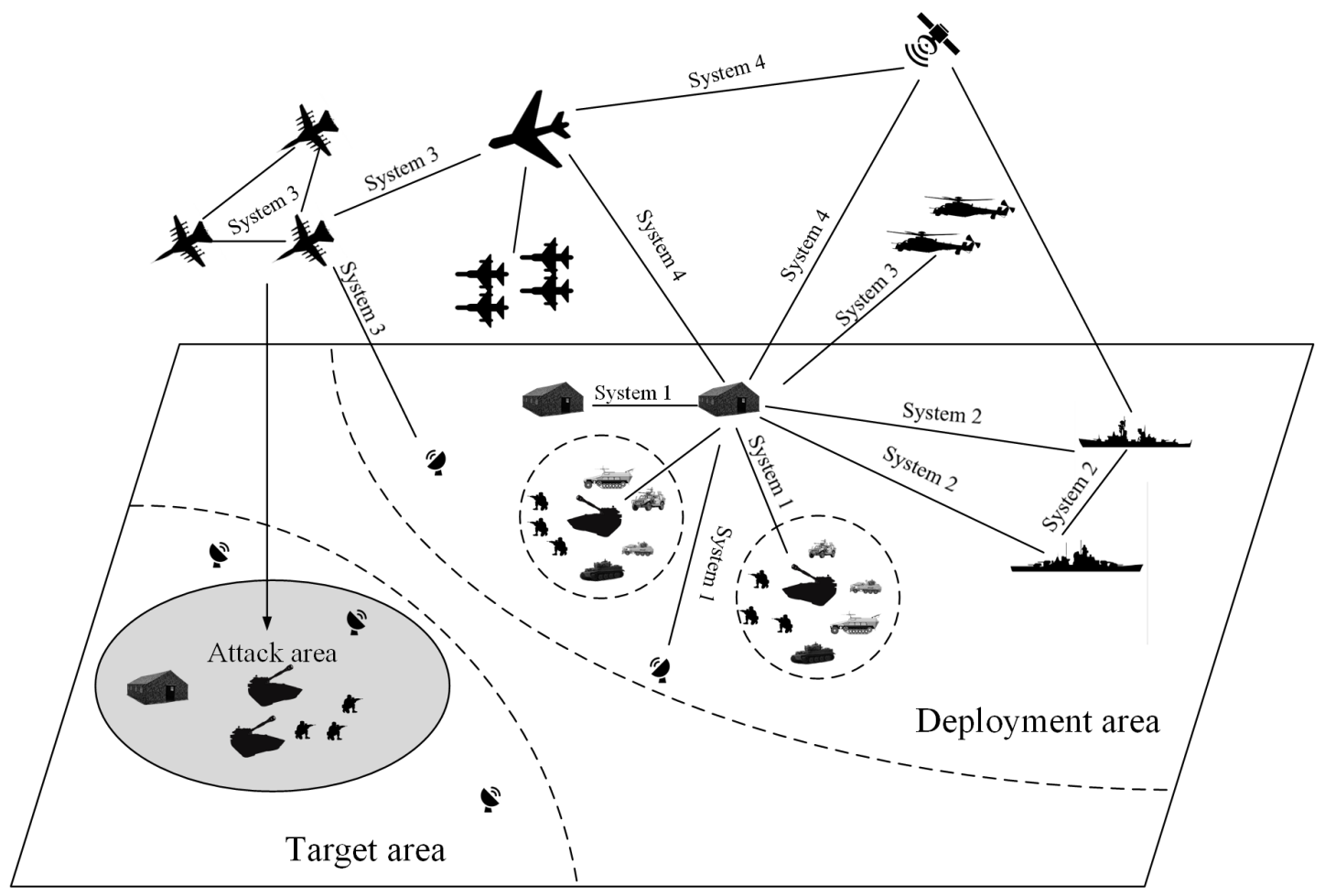

Figure 1. Multiple combat units with different information systems in the battlefield space.

In the process of operation, combat units are the basic units involved in combat tasks, and a combat mission can usually be accomplished through the cooperative action of multiple basic combat units. The battlefield situation information describes the observable states of these combat units in the battlefield space at a moment, including where they are, what they are doing, what status of movement they are in, what kind of relationships among them exist, and so on. Although information in different systems is heterogeneous, it is consistent from the aspect of content expressed in the information. No matter which system the heterogeneous information comes from, it essentially describes the information of categories, attributes, states, and inter-relationships owned by combat units. Thus, we take combat units as the core description objects to model the battlefield situation, which possesses the features of its nature, easy understanding, and close to reality.

\subsection{Entity Oriented Formal Representation of the Battlefield Situation}

To simulate combat units effectively, we adopted the entity oriented method, and entities were used to represent the independently identifiable, tangible geographical targets, combat units, and intangible concepts, such as mission or event, associated with combat operations. As shown in Figure 2, if we regard the physical space, i.e., the battlefield space, as a real space, and the information space composed of information describing the physical space as a virtual space, then we can take the entity in the virtual space as the mirror image of the corresponding target unit in the real space. A mapping relationship between entities and target units can be constructed. Then, the description information of entities in virtual space can correspondingly reflect the aspects such as the attributes or states of the target units in real space. Thus, we propose the concept of entity space, which is used to model the physical space. 
Definition 1 (entity space). Suppose $E S(t)=\left(E, C_{\mathrm{EC}}, A, S, R, V, \mathrm{~F}, t\right)$; where $\mathrm{E}$ represents the complete set of entities in the entity space; $C_{\mathrm{EC}}$ represents the collection of entity classes, which reflects the unary relationship of entities themselves in the entity space; $A$ describes the attributes of entities; $S$ describes the possible states of entities; $R$ describes the possible semantic relationships, which can be viewed as binary relationships among entities; $V$ represents the domain set of $A$ and $S$, while $\mathrm{V}=\mathrm{V}_{\mathrm{A}} \cup \mathrm{V}_{\mathrm{S}}$, and $\mathrm{V}_{\mathrm{A}}=\mathrm{U}_{\mathrm{a} \in \mathrm{A}} \mathrm{V}_{\mathrm{a}} \wedge \mathrm{V}_{\mathrm{S}}=\mathrm{U}_{\mathrm{s} \in \mathrm{S}} \mathrm{V}_{\mathrm{s}}$ is satisfied, where $\mathrm{V}_{\text {a }}$ represents the domain of attribute a and $\mathrm{V}_{s}$ represents the domain of state $\mathrm{s} ; \mathrm{F}$ represents the set of unary or pluralistic relational mappings from $A$ to $S$.

Suppose that in system $\mathbf{A}$, the number of attributes possessed by entity $e_{i}$ is $k$, and the number of state variables used to describe eiis $m$. Denote the value of the $j$ th attribute variable at time $t_{i}$ as $a_{i j}\left(t_{i}\right)$ and the value of the kth state variable at time $t$ as $s_{i k}\left(t_{i}\right)$. Then, the state set of $e_{i}$ at time $t_{i}$ can be described as:

$$
S_{i}\left(t_{i}\right)=\left\{s_{i 1}\left(t_{i}\right), s_{i 1}\left(t_{i}\right) \ldots, s_{i m}\left(t_{i}\right) \mid m \in \mathbf{N}\right\}=f_{i}\left(a_{i 1}\left(t_{i}\right), a_{i 2}\left(t_{i}\right), \ldots, a_{i k}\left(t_{i}\right)\right)
$$

where $f_{i}$ is a kind of relational mapping in $F$. If the information of categories, attributes, and states of each entity in $E$, as well as the information of relationships among entities are clarified, then an instance of $E S$ can be obtained, which is denoted as $I S_{A}\left(E S\left(t_{i}\right)\right)$. It reflects the state of the real battlefield situation at time $t_{i}$.

At any time $t$, the information in each information system describes an instance of the entity space respectively, where the instance of the entity space constructed based on the ith system is denoted as $I S_{i}\left(E S\left(t_{i}\right)\right)$. These instances correspondingly reflect an overall observable state of the battlefield space at that time, respectively. However, due to the different application requirements and information acquisition capabilities of different systems, the information of a single system usually describes some aspects of the physical space, but cannot describe the whole battlefield space; for example, as the reconnaissance scope and capability of reconnaissance equipment in a single system are limited, while various anti-reconnaissance means such as camouflage and interference are usually adopted by target combat units. Only partial attribute information of some external targets in a certain local area of the entire combat area can be obtained by the reconnaissance equipment.

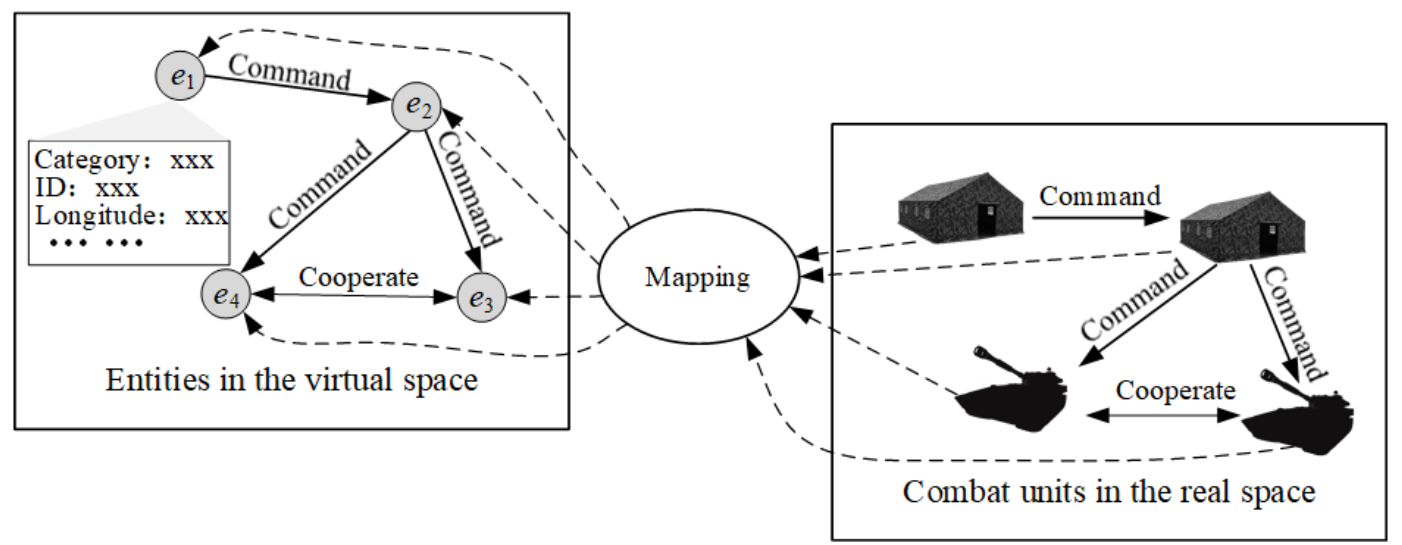

Figure 2. Entity oriented mapping from the real space to virtual space.

If the description information of the entity space in different systems can be aggregated and integrated, then a global entity space instance can be obtained, which can be formally described as:

$$
I S_{u}(E S(t))=\cup_{i}^{n} I S_{i}(E S(t))
$$

where $n$ represents the number of information systems used in the real space. If each combat unit can access the information in $I S_{u}(E S(t))$, then they can get a more comprehensive description of the whole entity space, so as to give full play to the overall use efficiency of information. 
Therefore, a heterogeneous information sharing framework based on a unified information space is designed.

\subsection{Heterogeneous Information Sharing Framework Based on Unified Information Space}

As shown in Figure 3, at any time $t$, each instance of the entity space, consisting of information from combat systems, can be viewed as a local battlefield information space (BSIS). Each BSIS describes a portion of the entire battlefield space, and they have both overlaps and differences with respect to the scope, as well as the content of the battlefield space described. On this condition, take entities as the basic units of information organization; the decentralized information transmitted in different systems can be organized and integrated. Meanwhile, a more comprehensive and complete information collection describing battlefield information, which is called the unified battlefield situation information space (UBSIS), can be obtained.

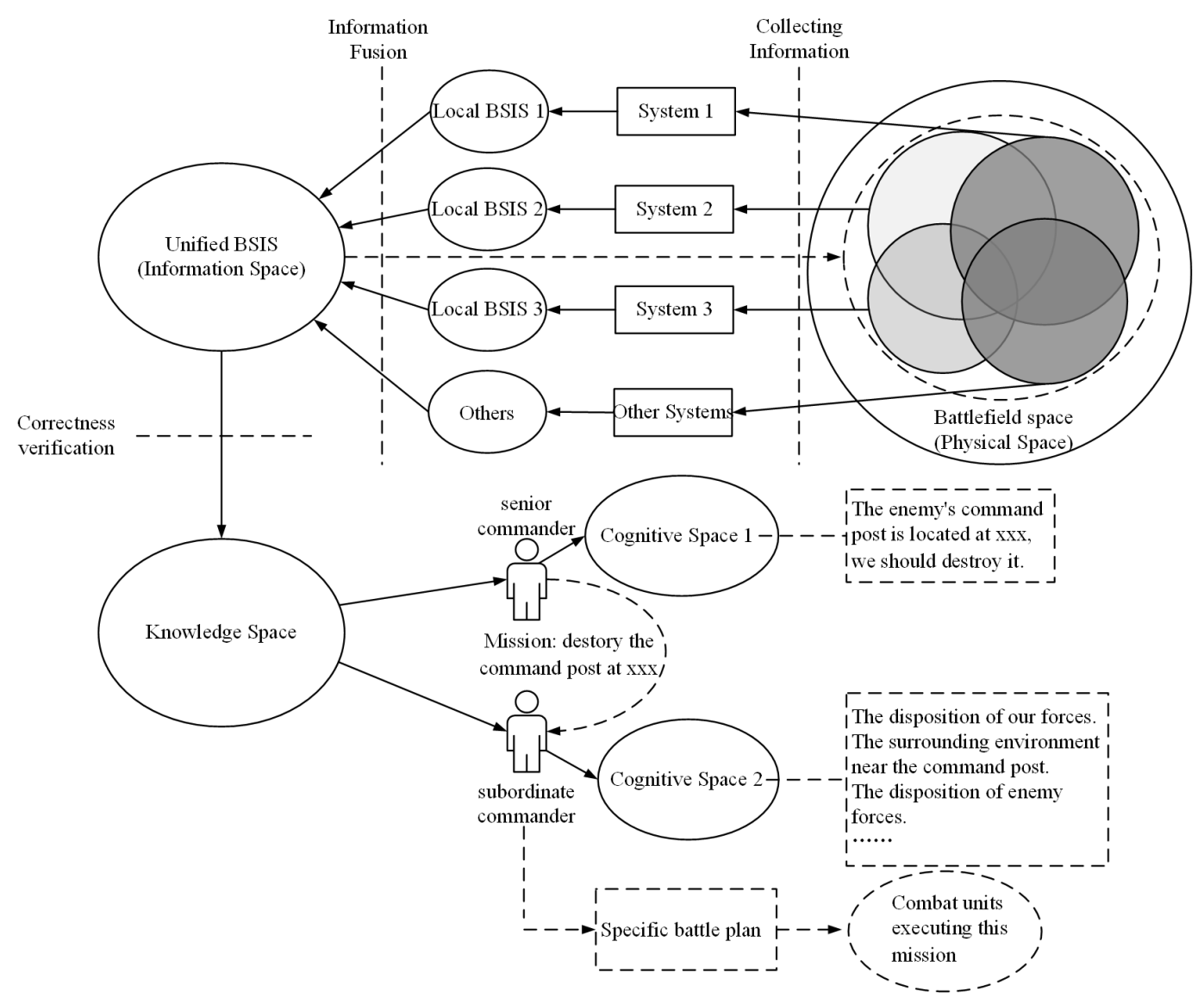

Figure 3. Flow of information based on the unified information space.

Based on UBSIS, a single system can capture more battlefield situation information than before, so that the system functions can be more effectively performed and more effective information services can be provided for combat units within the system. For example, the misjudgment of friend or foe, which is caused by possible errors in the identification algorithm and incomplete target information in a single system, can be reduced. Besides, commanders and other combat units can form a consistent understanding and cognition of the current battlefield situation based on the unified information space, which is conducive to provide better information support for distributed decision-making and to improve the effectiveness of joint operations. For example, when a commander makes a decision to attack a target, every combat unit in different systems can exactly know what and where the target is just as the commander knows and take corresponding action, which will improve the efficiency of the operation. 
Moreover, different combat units may need information about different aspects of the same target when they execute different missions. For example, when the senior commander makes a decision to attack a target, e.g., a command post, he/she may just need to know where the command post is; while the subordinate commander and combat units executing this mission need to know more detailed information, such as what is the surrounding environment near the command post, how many troops there are and their combat capability, etc. As the UBSIS can be viewed as a complete collection of information that describes the battlefield situation at a given moment, each combat unit can obtain the information on demand, which will be useful for realizing the distribution of information according to needs.

Based on the above analysis, we designed a layered heterogeneous information sharing framework, which is shown in Figure 4. The information transmission layer is mainly used to complete the generation and transmission of messages in each combat information system. It is the main body to realize transmission access, link control and data control, which provides control support and data transfer services to the upper level. The information presentation layer is used to realize the mapping between the proprietary message encoding format and the general information description format. The information sharing layer is mainly used to maintain the unified battlefield situation information space, which is shared by all combat units, and realize information query and information distribution.

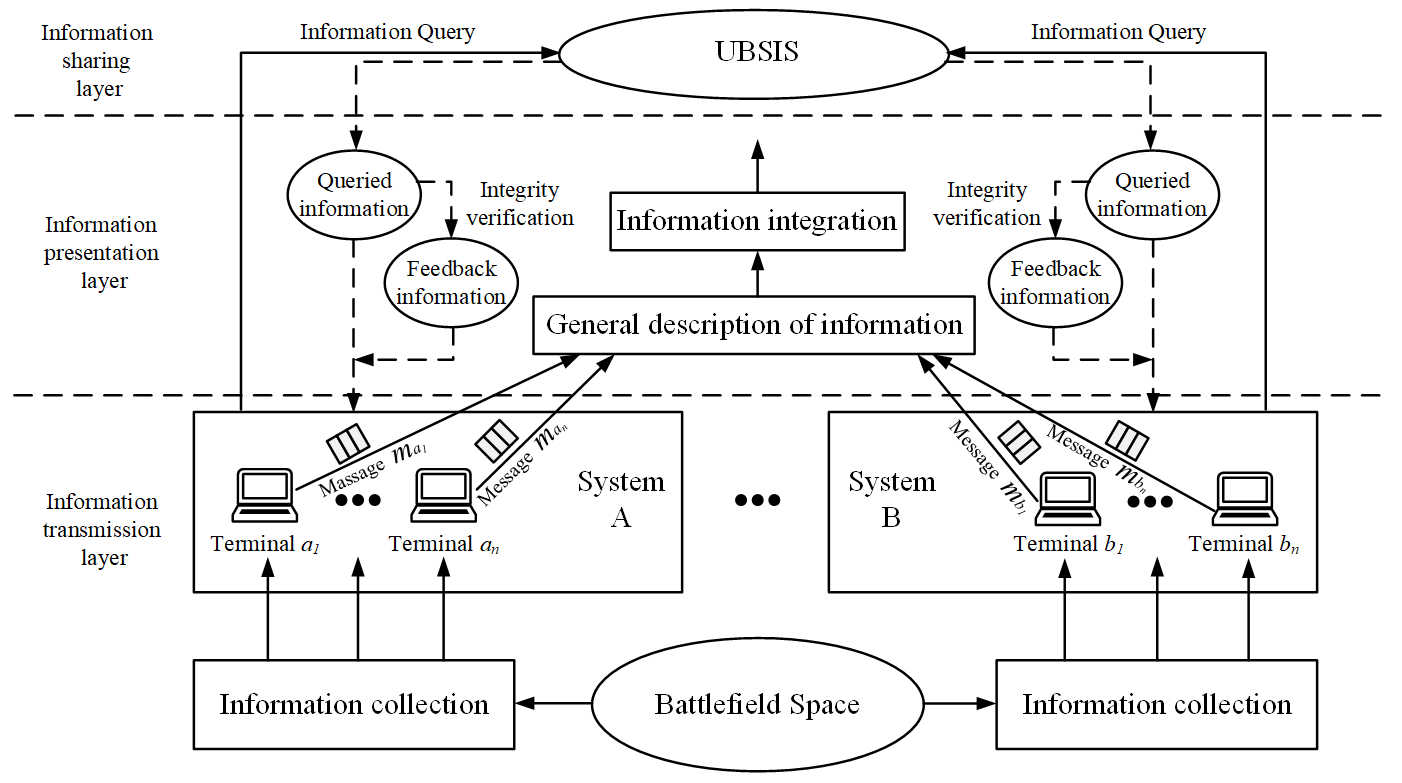

Figure 4. Schematic diagram of information sharing based on a unified information space.

To realize effective information sharing based on the proposed framework, we focused on the general description of heterogeneous information, the construction method of the unified battlefield situation information space, and the content based query mechanism.

\section{Entity Oriented General Description of Battlefield Situation Information}

In this section, we first introduce the structure of the concept-logic tree that is used to organize the concepts applied in describing the information of the battlefield situation. Then, we introduce the method of realizing the general description of heterogeneous information in detail.

\subsection{Structure of the Concept-Logic Tree}

As Figure 5 shows, some relations between $E, C_{E C}, A, S$, and $R$ exist. For example, the entity classes to which an entity belongs have relationships such as inclusion or intersection from the perspective of abstraction; the attributes of an entity are closely related to the entity classes that the entity belongs to; there are unary or multiple constraint relationships between attributes and states; a hierarchical 
structure and multi-semantic relations exist among entity relationships, and so on. Realizing a structured organization of concepts used in $E S(t)$ with these relations explicitly described will be useful. It can be used to realize a hierarchically structured organization of information in information systems and to improve the efficiency of information management and sharing among systems. Thus, we designed a concept-logic tree model to organize these concepts. Besides, to organize concepts in a structured way, we analyzed the logical and semantic relations between concepts that describe entities and constructed the concept-logic tree of both attributes and entity relationships, respectively.

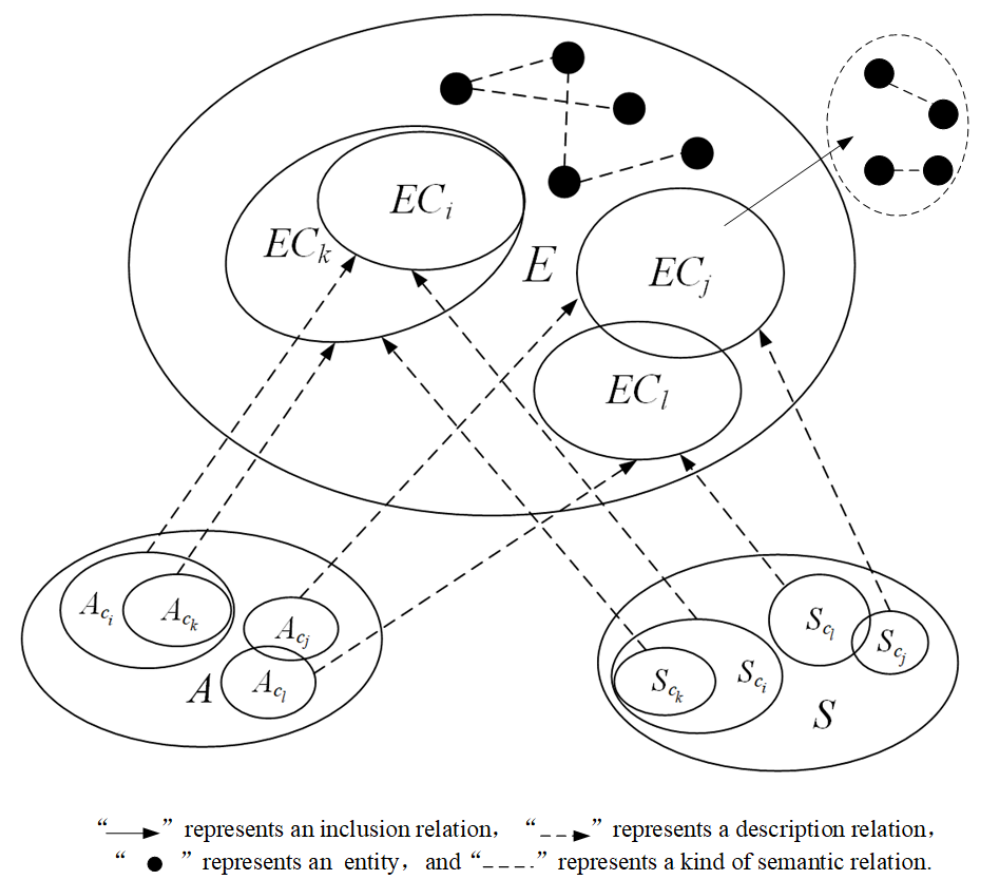

Figure 5. Relations among entities, entity classes, attributes, and states.

\subsubsection{Concept-Logic Tree of Attributes}

As attributes of entities are closely related to corresponding entity classes, to construct a structured description model of attributes, the logical hierarchical relationship of entity classes needs to be described first. Following are several basic relations among $C_{E C}$, and the reasoning rules based on them are shown in Table 2.

- Inclusion: If $\left\{E C_{i} \rightarrow A_{\mathcal{c}_{i} c}, E C_{j} \rightarrow A_{\mathcal{c}_{j} c}\right\} \wedge A_{\mathcal{c}_{i} c} \subseteq A_{\mathcal{c}_{j} c}$, then $E C_{i}$ include $E C_{j}$, which is denoted as $E C_{j} \subseteq E C_{i}$; while in this case, $E C_{j}$ is also called a subordinate class of $E C_{i}$.

- Independence: If $\left\{E C_{i} \rightarrow A_{c_{i} c}, E C_{j} \rightarrow A_{c_{j} c}\right\} \wedge A_{c_{i} c} \cap A_{c_{j} c}=\varnothing$, then $E C_{i}$ is independent of $E C_{j}$, which is denoted as $E C_{i} \perp E C_{j}$.

- Intersection: If $\left\{E C_{i} \rightarrow A_{c_{i} c}, E C_{j} \rightarrow A_{\mathcal{c}_{j} c}\right\} \wedge A_{c_{i} c} \cap A_{c_{j} c} \neq \varnothing \wedge\left\{A_{c_{i} c} \not A_{\mathcal{c}_{j} c}, A_{c_{c_{j}} c} \not A_{\mathcal{c}_{i}}\right\}$, then $E C_{i}$ and $E C_{j}$ intersect each other, which is denoted as $E C_{i} \chi E C_{j}$.

- Homology: If $E C_{i} \chi E C_{j} \wedge\left\{\exists E C_{k} \rightarrow A_{\mathcal{c}_{k} c} \wedge A_{\mathcal{c}_{k} c} \subseteq\left(A_{\mathcal{c}_{i} \mathcal{c}} \cap A_{\mathcal{c}_{j} c}\right)\right\}(k \in \mathbf{N} \wedge k \neq i \wedge k \neq j)$, then $E C_{i}$ and $E C_{j}$ are homogenous, which is denoted as $E C_{i} \bowtie E C_{j}$.

- Equivalence: If $\left\{E C_{i} \rightarrow A_{c_{i} c}, E C_{j} \rightarrow A_{c_{j} c}\right\} \wedge A_{c_{i} c}=A_{c_{j} c}$, then $E C_{i}$ is equal to $E C_{j}$, which is denoted as $E C_{i} \equiv E C_{j}$. 
Table 2. Reasoning rules based on the relationships among entity classes.

\begin{tabular}{|c|c|c|}
\hline Rule & Expression & Property \\
\hline 1 & IF $E C_{i} \subseteq E C_{j}, E C_{j} \subseteq E C_{k}$, THEN $E C_{i} \subseteq E C_{k}$ & Transitivity \\
\hline 2 & IF $E C_{j} \equiv E C_{i}, E C_{k} \equiv E C_{j}$, THEN $E C_{k} \equiv E C_{i}$ & Transitivity \\
\hline 3 & IF $E C_{j} \bowtie E C_{i}, E C_{k} \bowtie E C_{j}$, THEN $E C_{k} \bowtie E C_{i}$ & Transitivity \\
\hline 4 & 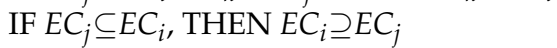 & Reversibility \\
\hline 5 & IF $E C_{j} \perp E C_{i}$, THEN $E C_{i} \perp E C_{j}$ & Symmetry \\
\hline 6 & $\operatorname{IF} E C_{j} \gamma E C_{i}$, THEN $E C_{i} \gamma E C_{j}$ & Symmetry \\
\hline 7 & IF $E C_{j} \equiv E C_{i}$, THEN $E C_{i} \equiv E C_{j}$ & Symmetry \\
\hline 8 & IF $E C_{j} \bowtie E C_{i}$, THEN $E C_{i} \bowtie E C_{j}$ & Symmetry \\
\hline 9 & IF $E C_{j} \subseteq E C_{i}, E C_{i} \subseteq E C_{j}$, THEN $_{E C_{i}} \equiv E C_{j}$ & Implication \\
\hline 10 & IF $E C_{i} \subseteq E C_{j}, E C_{j} \perp E C_{k}$, THEN $E C_{i} \perp E C_{k}$ & Implication \\
\hline 11 & IF $E C_{i} \subseteq E C_{j}, E C_{j} \bowtie E C_{k}$, THEN $E C_{i} \bowtie E C_{k}$ & Implication \\
\hline 12 & IF $E C_{i} \equiv E C_{j}, E C_{j} \perp E C_{k}$, THEN $E C_{i} \perp E C_{k}$ & Implication \\
\hline 13 & IF $E C_{i} \equiv E C_{j}, E C_{j} \gamma E C_{k}$, THEN $E C_{i} \chi E C_{k}$ & Implication \\
\hline 14 & IF $E C_{i} \equiv E C_{j}, E C_{j} \subseteq E C_{k}$, THEN $E C_{i} \subseteq E C_{k}$ & Implication \\
\hline 15 & IF $E C_{i} \equiv E C_{j}, E C_{j} \bowtie E C_{k}$, THEN $E C_{i} \bowtie E C_{k}$ & Implication \\
\hline
\end{tabular}

Typically, we can use a tree structure to describe these relationships among entity classes explicitly, i.e., a classification tree of entity classes can be built based on the relationships among entity classes.

Definition 2 (entity class classification tree). Suppose $C T_{\mathrm{EC}}\left(V_{\mathrm{EC}}\right)$ is a tree with $V_{\mathrm{EC}}$ as the root node and all entity classes in the research field as its child nodes, while the nodes are organized by the logical relationships among entity classes.

Figure 6 displays the basic structure of $C T_{E C}\left(V_{E C}\right)$, where $V_{E C}$ can be viewed as a virtual entity class, which is used to represent the class with the highest degree of abstraction. The level of $V_{E C}$ is assumed to be zero, and no actual attribute set or state set is attached to it. Besides, the attribute set of $V_{E C}$ is denoted as $A_{E C}$, which contains only the attributes that are common to all entities in the entity space such as the time-attribute and space-attribute. The state set of $V_{E C}$ is denoted as $S_{E C \text {, which is }}$ regarded to be empty or only contains the state of "exist".

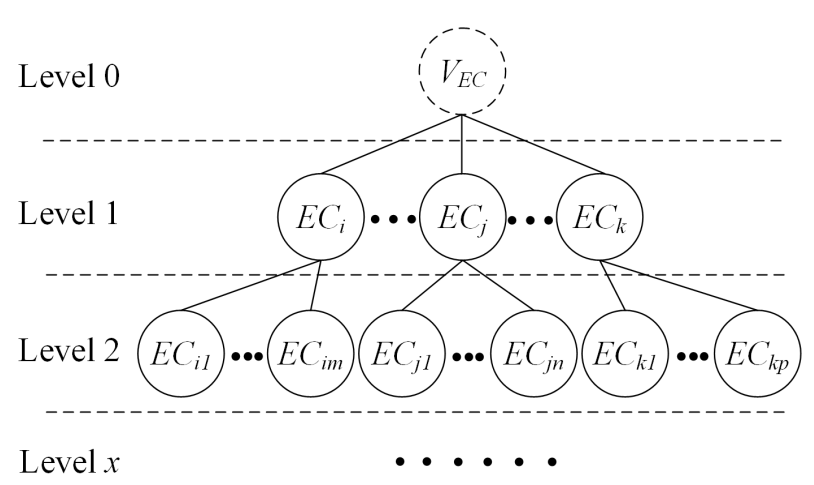

Figure 6. Structure of the entity class classification tree.

Based on the structure of the entity class classification tree and the property inheritance relationship between the parent and the subclass, the attribute concept-logic tree can be obtained.

Definition 3 (attribute concept-logic tree). The attribute concept-logic tree is denoted as $T_{\mathrm{LEC}}\left(E C_{\mathrm{i}}\right)$, which is a tree with $E C_{\mathrm{i}}$ as the root node, and can be formally expressed as $T_{\mathrm{LEC}}\left(E C_{\mathrm{i}}\right)=\left(E C_{\mathrm{i}}\left(S_{\mathrm{C}_{\mathrm{i}}}^{\prime} \cup{S_{\mathrm{C}_{\mathrm{i}} \mathrm{O}}}^{\prime}, A_{\mathrm{C}_{\mathrm{i}} \mathrm{O}}^{\prime}, A_{\mathrm{C}_{\mathrm{i}}}^{\prime}\right.\right.$ $\left.\cup T_{L E C}\left(E C_{\mathrm{j}}\right)\right)$ ), where $i, j \in N$, and $E C_{\mathrm{j}} \in C_{\mathrm{EC}_{\mathrm{i}}} . A_{\mathrm{c}_{\mathrm{i}} \mathrm{O}}^{\prime}$ is obtained by stratifying the occasional attribute set according to the entity class classification tree, e.g., if $E C_{\mathrm{j}}$ is the direct subordinate class of $E C_{\mathrm{i}}$, then $A_{\mathrm{C}_{\mathrm{i}} \mathrm{O}}^{\prime}=A_{\mathrm{c}_{\mathrm{j}} \mathrm{O}}$ $-A_{\mathrm{c}_{\mathrm{i}} \mathrm{O}}$. Figure 7 shows the basic structure of $T_{\mathrm{LEC}}\left(E C_{\mathrm{i}}\right)$. 


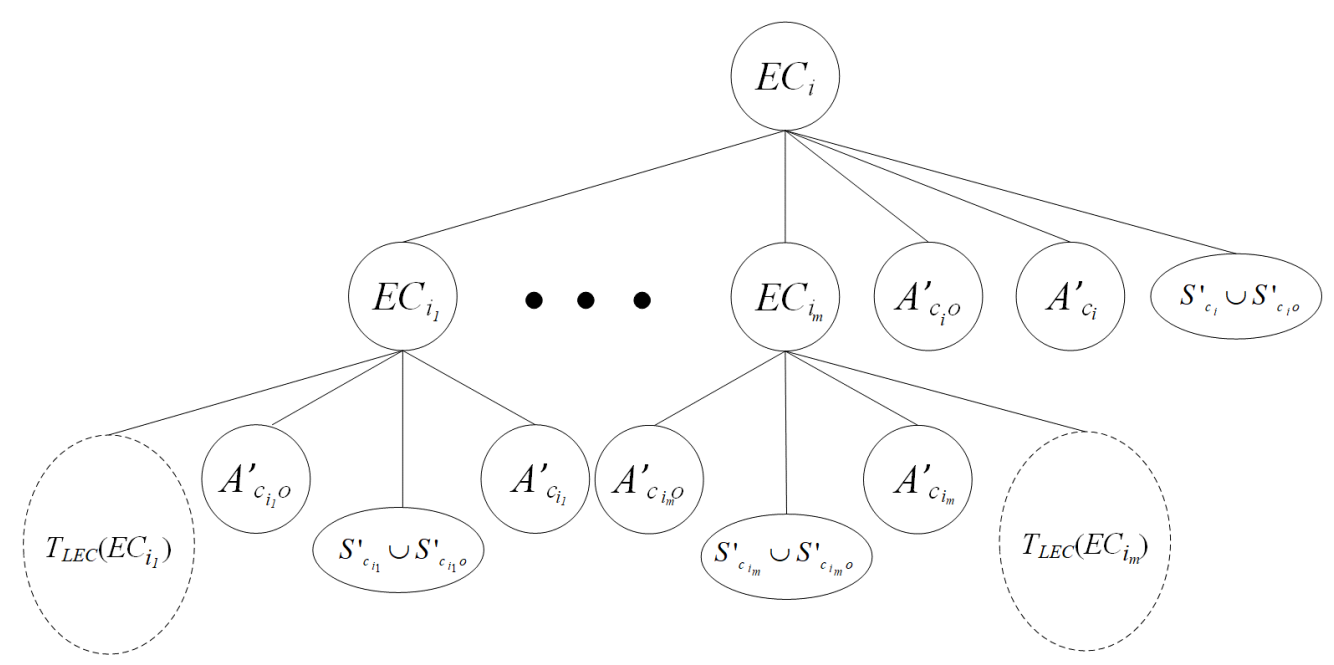

Figure 7. Structure of the entity class classification tree.

\subsubsection{Concept-Logic Tree of Entity Relationships}

Take $r_{a}, r_{b}$, and $r_{c}$ as three kinds of entity relationships, then several main relations among entity relationships can be described as follows; where entity relationships are directed, e.g., $e_{x} \stackrel{r_{a}}{\rightarrow} e_{y}$ means an entity $e_{x}$ is connected with another entity $e_{y}$ through relationship $r_{a}$.

- Inclusion: If $e_{x} \stackrel{r_{a}}{\rightarrow} e_{y} \Rightarrow e_{x} \stackrel{r_{b}}{\rightarrow} e_{y}$, then $r_{b}$ include $r_{a}$, which is denoted as $r_{a} \subseteq r_{b}$.

- Metex: If $e_{x} \stackrel{r_{a}}{\rightarrow} e_{y} \Rightarrow e_{x} \stackrel{\neg r_{b}}{\longrightarrow} e_{y}$, then $r_{a}$ and $r_{b}$ are mutually exclusive, e.g., $r_{a}$ and $r_{b}$ cannot exist between two entities at the same time, which is denoted as $r_{a} \perp r_{b}$.

- Compatibility: If $r_{a}$ and $r_{b}$ can exist between two entities at the same time, then $r_{a}$ and $r_{b}$ are compatible, which is denoted as $r_{a} \| r_{b}$.

- Inverse: If $e_{x} \stackrel{r_{a}}{\rightarrow} e_{y} \Rightarrow e_{y} \stackrel{r_{b}}{\rightarrow} e_{x}$, then $r_{a}$ and $r_{b}$ possess the inverse relationship, which is denoted as $r_{a}^{-1}=r_{b}$. Since the entity relationship describes the mutual relation between two entities, there must be an inverse relation $r_{b}$ for any $r_{a}$. At this time, the semantics expressed by $e_{x} \stackrel{r_{a}}{\rightarrow} e_{y}$ and $e_{y} \stackrel{r_{b}}{\rightarrow} e_{x}$ are equivalent. In particular, if $r_{a}^{-1}=r_{a}$, then $r_{a}$ is considered to be symmetric.

- Accessibility: If $e_{x} \stackrel{r_{a}}{\rightarrow} e_{y} \wedge e_{y} \stackrel{r_{b}}{\rightarrow} e_{z} \Rightarrow e_{x} \stackrel{r_{c}}{\rightarrow} e_{z}$, then $r_{a}$ and $r_{c}$ are accessible, which is denoted as $r_{a}$ $\rightleftarrows r_{c}$ or $r_{a} \bullet r_{b}=r_{c}$.

From the perspective of semantic expression, entity relationships reflect the semantic connections between entities. Thus, when judging the relations among entities, the logic of semantics expressed by entity relationships is mainly considered. For example, when describing the relationship among combat units, if $e_{x} \stackrel{r_{a}}{\rightarrow} e_{y}$ means $e_{x}$ command $e_{y}, e_{y} \stackrel{r_{b}}{\rightarrow} e_{x}$ means $e_{y}$ is commanded by $e_{x}$, and $e_{x} \stackrel{r_{c}}{\rightarrow} e_{z}$ means $e_{x}$ collaborate with $e_{z}$, then $r_{a}$ and $r_{b}$ possess a reciprocal relation, while $r_{b}$ and $r_{c}$ are compatible. Clarifying the hierarchy between entity relationships is conducive to further clarifying the reasoning rules among entity relationships and to realize semantics based reasoning on the basis of obtaining entity description information. Table 3 shows some reasoning rules based on entity relationships. 
Table 3. Reasoning rules based on entity relationships.

\begin{tabular}{clc}
\hline Rule & Expression & Property \\
\hline 1 & IF $r_{a} \subseteq r_{b}, r_{b} \subseteq r_{c}$, THEN $r_{a} \subseteq r_{c}$ & Transitivity \\
2 & IF $r_{a} \rightleftarrows r_{b}, r_{b} \rightleftarrows r_{c}$, THEN $r_{a} \rightleftarrows r_{c}$ & Transitivity \\
3 & IF $r_{a} \subseteq r_{b}$, THEN IF $r_{b} \supseteq r_{a}$ & Reversibility \\
4 & IF $r_{a}^{-1}=r_{b}$, THEN $r_{b}^{-1}=r_{a}$ & Symmetry \\
5 & IF $r_{a} \perp r_{b}$, THEN $r_{b} \perp r_{a}$ & Symmetry \\
6 & IF $r_{a} \| r_{b}$, THEN $r_{b} \| r_{a}$ & Symmetry \\
7 & IF $r_{a} \rightleftarrows r_{b}$, THEN $r_{b} \rightleftarrows r_{a}$, & Symmetry \\
8 & IF $r_{a} \subseteq r_{b}, r_{b} \perp r_{c}$, THEN $r_{a} \perp r_{c}$ & Implication \\
9 & IF $r_{a} \subseteq r_{b}, r_{b} \| r_{c}$, THEN $r_{a} \| r_{c}$ & Implication \\
10 & IF $r_{a} \| r_{b}, r_{a}^{-1}=r_{c}$, THEN $r_{c} \| r_{b}$ & Implication \\
11 & IF $r_{a}^{-1}=r_{b}, r_{b}^{-1}=r_{c}$, THEN $r_{a}=r_{c}$ & Implication \\
12 & IF $r_{a} \bullet r_{b}=r_{c}$, THEN $r_{c} \bullet r_{b}^{-1}=r_{a}^{-1}$ & Implication \\
\hline
\end{tabular}

Based on the above relationships, the concept of the entity relationship concept-logic tree is proposed, which organizes the entity relationships hierarchically according to the logical and semantic relations among entity relationships.

Definition 4 (entity relationship concept-logic tree). Suppose $T_{\mathrm{LEC}}(\mathrm{Fr})$ is a tree with Fr as the root node, and other entity relationships defined in the research field are organized as child nodes of Fr, according to the logical and semantic relations among entity relationships. In this tree, Fr represents an entity relationship with the highest abstraction degree.

Generally, the concept-logic model can be viewed as a concept model, which organizes the concepts describing entity information in a structured way and is further used to realize the consistent description of information. Obviously, in order to construct a general concept-logic model to realize heterogeneous information integration, the concepts used in it should be selected based on the consensus of researchers in field of study. The mapping of concepts from the general concept-logic model to that used in each system should be designed. This makes the global entity space model effectively cover the information expression range of different systems, i.e., the local entity space model constructed based on each system is consistent with the global entity space model.

\subsection{Description and Organization of Internal Information about Entities Based on a Template}

The internal information about entities refers to the static information such as the category, attribute, and state of entities.

For this information, we designed a general information description template based on the attribute concept-logic tree to realize the uniform description and organization of the heterogeneous information from different systems. When designing templates, two aspects should be paid attention to: (1) selecting the appropriate information description language to ensure the accuracy, sufficiency, and flexibility of information description; (2) selecting the appropriate template structure to ensure the consistency of the heterogeneous information description.

As a widely used markup language currently, XML has the advantages of self-description, structure, and platform independence, as well as strong knowledge representation ability, semantic annotation ability, and extensibility. Therefore, we adopted XML as the description language in the design of the general information description templates, i.e., templates were constructed with XML and stored as XML documents. Considering that an XML document is formally represented as a tree structure after parsing, which has a good comparison relationship with the attribute concept-logic tree model, we adopted a tree structure to design the general information description templates. Besides, to ensure the integration correctness and efficiency of the presented information, when designing a template, the structure of the template was in strict accordance with the structure of the corresponding 
attribute concept-logic tree. That is, one-to-one correspondence between the nodes of the attribute conceptual logical tree and the nodes of the template parse tree existed.

As showed in Figure 8, based on the general information description templates, the heterogeneous internal information about entities can be described, organized, and stored in a structured way; where the information organized is stored with XML documents that are entity oriented, i.e., the information of each entity is stored with a document separately. On this basis, operations such as information integration and acquisition can be transformed into the corresponding operations such as pruning, merging, or retrieval based on the information tree, which is easy to implement.

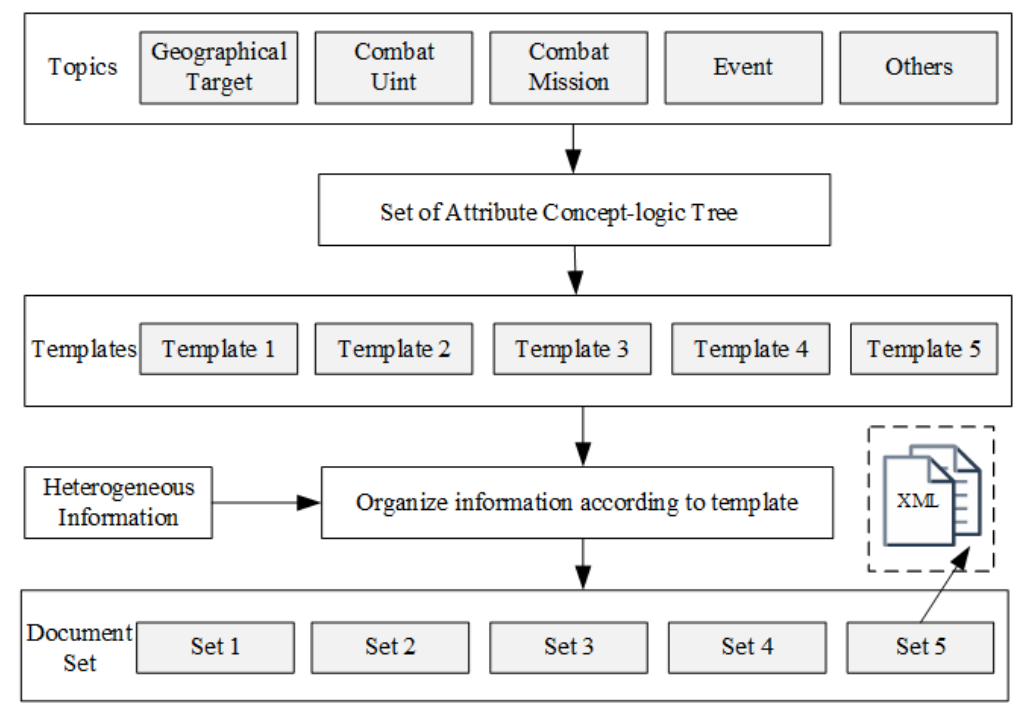

Figure 8. Structure of the entity class classification tree.

\subsection{Description and Organization of External Information about Entities Based on the Semantic Link Network}

Entity relationships are used to describe the relations between geographic targets, combat units, and concepts such as combat missions. Among them, the relations among geographic targets are stable and can be viewed as static within a somewhat long period. The relations between the combat unit and geographic target are relatively simple; they mainly describe the location and direction of combat units with respect to a particular geographic target in the combat area; while the relations among combat units are relatively complex, e.g., there are various relations exist, such as subordination, command, collaboration, guarantee, and so on. Since combat units possess complex attributes such as mobility, combat capability, and functionality, the relations among combat units are dynamic and mission related. For example, suppose $e_{x}, e_{y}$, and $e_{z}$ represent three combat units that belong to different services, while they are executing the same mission at time $t$. If $e_{x}$ possesses the command authority, then $e_{x} \stackrel{\text { Command }}{\longrightarrow} e_{y}, e_{x} \stackrel{\text { Command }}{\longrightarrow} e_{z}$, and $e_{z} \stackrel{\text { Collaborate }}{\longrightarrow} e_{y}$ are satisfied at this time. However, after the mission is finished, these relationships may no longer be satisfied. Thus, the relationships among entities actually form a relational network that is dynamic, real time, and mission related. To describe this relational network, we constructed a semantic link network of entity relationships.

The semantic link network is a self-organized semantic network that consists of semantic nodes, semantic links, and rules on semantic links; where a semantic space defines the semantics of semantic nodes, semantic links, and rules [13-15], and the interactive semantics and the semantic base were proposed for establishing the semantic basis [16]. The was originally used to support intelligent web applications by extending hyperlinks $[17,18]$, and the basic theory was developed systematically $[19,20]$. Currently, it is extended to simulate the basic structure of a cyber-physical society [21-23].

Thus, to describe the relationships among entities effectively, we constructed a semantic link network, which is denoted as $\langle N, L, R l>$; where $N$ represents the collection of semantic nodes, i.e., the entities existing in the entity space, $L$ represents the collection of semantic links, i.e., the relationships 
between entities, and $R l$ represents the collection of reasoning rules based on $L$, which is closely related to the properties of entity relationships. When storing the information of entity relationships in an SLN, an adjacency matrix called the Semantic Relationship Matrix (SRM) is used. For example, suppose there are $n$ entities $\left\{e_{1}, e_{2}, \ldots, e_{n} \mid n \in \mathbf{N}\right\}$ in the entity space, then the corresponding SLN can be represented by an SRM as follows.

$$
\left(\begin{array}{cccc}
r_{11} & r_{12} & \ldots & r_{1 n} \\
r_{21} & r_{22} & \ldots & r_{2 n} \\
\ldots & \ldots & \ldots & \ldots \\
r_{n 1} & r_{n 2} & \ldots & r_{n n}
\end{array}\right)
$$

where $r_{i j}(i, j \in[1, n])$ represents the semantic links from $e_{i}$ to $e_{j}, r_{i i}=e_{i}$, and $r_{i j}=r_{j i}^{-1}$. If there are no semantic links between $e_{i}$ and $e_{j}$, then $r_{i j}=r_{j i}^{-1}=$ Null.

Generally, an element in SRM should be a set of semantic indicators as there may be multiple semantic links between the same pair of nodes, and a three-dimensional tensor can be used to represent a semantic link network [24,25]. Moreover, since semantic links are dynamically evolved, based on SRM and the reasoning rules in $R l$, the semantic links between any two entities can be obtained, which is useful in the search for entities meeting specific semantic constraints.

\section{Construction of the Unified Battlefield Situation Information Space}

The unified battlefield situation information space (UBSIS) is the virtual space constituted by the information of each combat information system, that is the information space obtained after the entity description information in each system is described consistently and stored centrally. The internal description information about entities is stored in the XML documents in an entity oriented manner, and the external information about entities is stored in the semantic relationship matrix. In the process of constructing and updating the UBSIS, the information describing the same entity needs to be aggregated so as to eliminate information redundancy effectively. The way of realizing the aggregation of the internal and external description information about entities is introduced below, respectively.

\subsection{Aggregation of Internal Description Information about Entities}

Description information about entities is usually carried by the messages delivered within systems, and then, the process of information aggregation can be viewed as the process of aggregating message content.

Suppose $m_{i}$ is a piece of message that describes the information of $e_{i}$ belonging to $E C_{i}$. Use $T M$ $\left(m_{i}\right)$ to denote the tree parsed from $\operatorname{Doc}\left(m_{i}\right)$ that is generated after decoding and structured operation of message $m_{i}$ and $T\left(e_{i}\right)$ to denote the parse tree of $\operatorname{Doc}\left(e_{i}\right)$ that stores the description information of $e_{i}$. As can be seen from Definition 3, when $e_{i}$ is completely described, the tree structure formed by all the concepts involved should be a sub-tree of $T_{L E C}\left(E C_{i}\right)$, then we can get the following Equation (3), where $S_{c_{i+n}}^{\prime \prime}=S_{c_{i+n}}^{\prime} \cup S_{c_{i+n} 0}^{\prime} \wedge A_{c_{i+n}}^{\prime \prime}=A_{c_{i+n}}^{\prime} \cup A_{c_{i+n}}^{\prime}$ :

$$
T\left(e_{i}\right)=\left(E C_{i}\left(E C_{i+1}\left(\ldots\left(E C_{i+n}\left(S_{c_{i+n}}^{\prime \prime}, A_{c_{i+n}}^{\prime \prime}\right), S_{c_{i+n-1}}^{\prime \prime}, A_{c_{i+n-1}}^{\prime \prime}\right) \ldots\right), S_{c_{i}}^{\prime \prime}, A_{c_{i}}^{\prime \prime}\right)\right)(n \in \mathbf{N}) .
$$

Let the collection of messages describing $e_{i}$ from $\mathbf{A}$ and $\mathbf{B}$ be $M_{i}=\left\{m_{1}, m_{2}, \ldots, m_{q} \mid q \in \mathbf{N}\right\}$, then the tree structure obtained after information aggregation should be the same as $T\left(e_{i}\right)$. Denote the initial information tree structure describing $e_{i}$ in the description document of $e_{i}$ in UISas $T_{0}^{\prime}\left(e_{i}\right)$. Assume that no message describing $e_{i}$ has been received at present and any subsequent message describing $e_{i}$ is correct, i.e., it satisfies the constrains of logic and semantics between attributes and states that describe a specific entity. When the first message $m_{1}$ describing $e_{i}$ arrives, $T_{M}\left(m_{i}\right)$ is then the initial information tree structure of $e_{i}$, which is denoted as $T_{1}^{\prime}\left(e_{i}\right)$. Then, we can get Equation (4):

$$
T_{1}^{\prime}\left(e_{i}\right)=T_{M}\left(m_{1}\right)=\left(E C_{i}\left(E C_{i+1}\left(\ldots\left(E C_{i+k_{1}}\left(P_{1}^{\prime} S_{c_{i+k_{1}}}^{\prime \prime}, P_{1}^{\prime} A_{c_{i+k_{1}}}^{\prime \prime}\right), P_{1}^{\prime} S_{c_{i+k_{1}-1}}^{\prime \prime}, P_{1}^{\prime} A_{c_{i+k_{1}-1}}^{\prime \prime}\right) \ldots\right), P_{1}^{\prime} S_{c_{i_{i}}}^{\prime \prime} P_{1}^{\prime} A_{c_{i}}^{\prime \prime}\right)\right)
$$


where $k_{1} \leq n \wedge\left\{P_{1}^{\prime} S_{c_{j}}^{\prime \prime} \subseteq S_{c_{j^{\prime}}}^{\prime \prime}, P_{1}^{\prime} A_{c_{j}}^{\prime \prime} \subseteq A_{c_{j}}^{\prime \prime} \mid j \in[i, i+n]\right\}$.

When the second message $m_{2}$ arrives, Equation (5) holds:

$$
T_{M}\left(m_{2}\right)=\left(E C_{i}\left(E C_{i+1}\left(\ldots\left(E C_{i+k_{2}}\left(P_{2} S_{c_{i+k_{2}}}^{\prime \prime}, P_{2} A_{c_{i+k_{2}}}^{\prime \prime}\right), P_{2} S_{c_{i+k_{2}-1}}^{\prime \prime}, P_{2} A_{c_{i+k_{2}-1}}^{\prime \prime}\right) \ldots\right), P_{2} S_{c_{i}}^{\prime \prime}, P_{2} A_{c_{i}}^{\prime \prime}\right)\right)
$$

where $k_{2} \leq n \wedge\left\{P_{2} S_{c_{j}}^{\prime \prime} \subseteq S_{c_{j}}^{\prime \prime}, P_{2} A_{c_{j}}^{\prime \prime} \subseteq A_{c_{j}}^{\prime \prime} \mid j \in[i, i+n]\right\}$. Suppose $k_{2} \geq k_{1}$, then $T_{2}^{\prime}\left(e_{i}\right)=T_{1}^{\prime}\left(e_{i}\right) \oplus T_{M}\left(m_{2}\right)$, and we get Equation (6), where the " $\oplus$ " operator represents the operation of tree merge:

$$
T_{2}^{\prime}\left(e_{i}\right)=\left(E C_{i}\left(E C_{i+1}\left(\ldots\left(E C_{i+k_{2}}\left(P_{2}^{\prime} S_{c_{i+k_{1}}}^{\prime \prime}, P_{2}^{\prime} A_{c_{i+k_{2}}}^{\prime \prime}\right), P_{2}^{\prime} S_{c_{i+k_{2}-1}}^{\prime \prime}, P_{2}^{\prime} A_{c_{i+k_{2}-1}}^{\prime \prime}\right) \ldots\right), P_{2}^{\prime} S_{c_{i}}^{\prime \prime} P_{2}^{\prime} A_{c_{i}}^{\prime \prime}\right)\right)
$$

where $\left\{P_{2}^{\prime} S_{c_{j}}^{\prime \prime}=P_{2} S_{c_{j}}^{\prime \prime} \cup P_{1}^{\prime} S_{c_{j^{\prime}}}^{\prime \prime}, P_{2}^{\prime} A_{c_{j}}^{\prime \prime}=P_{2} A_{c_{j}}^{\prime \prime} \cup P_{1}^{\prime} A_{c_{j}}^{\prime \prime} \mid j \in\left[i, i+k_{2}\right]\right\} \wedge\left\{P_{1}^{\prime} S_{c_{l}}^{\prime}=\varnothing, P_{1}^{\prime} A_{c_{l}}^{\prime}=\varnothing \mid l \in\left(i+k_{1}, i+k_{2}\right]\right\}$.

By that analogy, when message $m_{g}$ arrives, $T_{g}^{\prime}\left(e_{i}\right)=T_{g-1}^{\prime}\left(e_{i}\right) \oplus T_{M}\left(m_{g}\right)$, and we get Equation (7)

$$
T_{g}^{\prime}\left(e_{i}\right)=\left(E C_{i}\left(E C_{i+1}\left(\ldots\left(E C_{i+k_{g}}\left(P_{g}^{\prime} S_{c_{i+k g}}^{\prime \prime}, P_{g}^{\prime} A_{c_{i+k g}}^{\prime \prime}\right), P_{g}^{\prime} S_{c_{i+k g}-1}^{\prime \prime}, P_{g}^{\prime} A_{c_{i+k g}-1}^{\prime \prime}\right) \ldots\right), P_{g}^{\prime} S_{c_{i}}^{\prime \prime}, P_{g}^{\prime} A_{c_{i}}^{\prime \prime}\right)\right)
$$

where, $\left\{P_{g}^{\prime} S_{c_{j}}^{\prime \prime}=P_{g} S_{c_{j}}^{\prime \prime} \cup P_{g-1}^{\prime} S_{c_{j}}^{\prime \prime} P_{g}^{\prime} A_{c_{j}}^{\prime \prime}=P_{g} A_{c_{j}}^{\prime \prime} \cup P_{g-1}^{\prime} A_{c_{j}}^{\prime \prime} \mid j \in\left[i, i+k_{g}\right]\right\} \wedge\left\{P_{g-1}^{\prime} S_{c_{l}}^{\prime \prime}=\varnothing, P_{g-1}^{\prime} A_{c_{l}}^{\prime \prime}=\varnothing \mid l\right.$ $\left.\in\left(i+k_{g-1}, \mathrm{i}+k_{g}\right]\right\}$.

If $\forall j \in[i, i+n], P_{g}^{\prime} S_{c_{j}}^{\prime}=S_{c_{j}}^{\prime}$ and $P_{g}^{\prime} A_{c_{j}}^{\prime}=A_{c_{j}}^{\prime}$ are satisfied, then $T_{g}^{\prime}\left(e_{i}\right)=T\left(e_{i}\right)$ can be obtained.

From the above analysis, it can be seen that the process of information fusion is actually the process of constantly updating and expanding the information tree describing $e_{i}$ in the UIS and ultimately making the information describing $e_{i}$ complete, i.e., all the information describing the states and attributes of $e_{i}$ have specific values. Subsequent messages are a partial replacement process for the existing information of a certain state or attribute. Therefore, information can be fused based on the concept-logic tree so as to reduce information redundancy, which is useful to eliminate the information search time.

\subsection{Aggregation of External Description Information about Entities}

As for the aggregation of external information about entities, it can be initially realized by the merge of semantic relationship matrices constructed from different combat information systems separately, which is denoted as $S R M_{u}=\cup_{i}^{n} S R M_{i}$.

The process of realizing the merger of semantic relationship matrices is as shown in Figure 9, and the main steps are as follows:

- Get $S R M_{i}$ from the $i$ th combat information system $i \in(0, n]$, and a collection of SRMs can be obtained, which is denoted as $C_{S R M}$.

- Get the corresponding $S R M_{i}^{+}$of $S R M_{i}$ by the general description of $S R M_{i}$ based on the entity relationship concept-logic tree, and then, $C_{S R M}^{+}$can be obtained.

- Get the combined semantic relationship matrix $S R M_{u}^{+}$by merging the matrices contained in $C_{S R M}^{+}$.

- Based on the reasoning rules in $R l$, realize the reasoning on semantic relationships between entities in $S R M_{u}^{+}$, and an extended semantic relation matrix of $S R M_{u}^{+}$can be obtained, which is denoted as $S R M_{u}^{\prime+}$.

$S R M_{u}^{\prime+}$ is the final result of realizing the merge of SRMs, and it describes the complete set of entity relationships between the entities in UBSIS. After that, by the evolution of $S R M_{u}^{\prime+}$, the newly obtained external information of $e_{i}$ can be integrated with that contained in $S R M_{u}^{\prime+}$, which reflects the operations such as add node, delete node, add edge, or delete edge related to $e_{i}$ in SLN. 


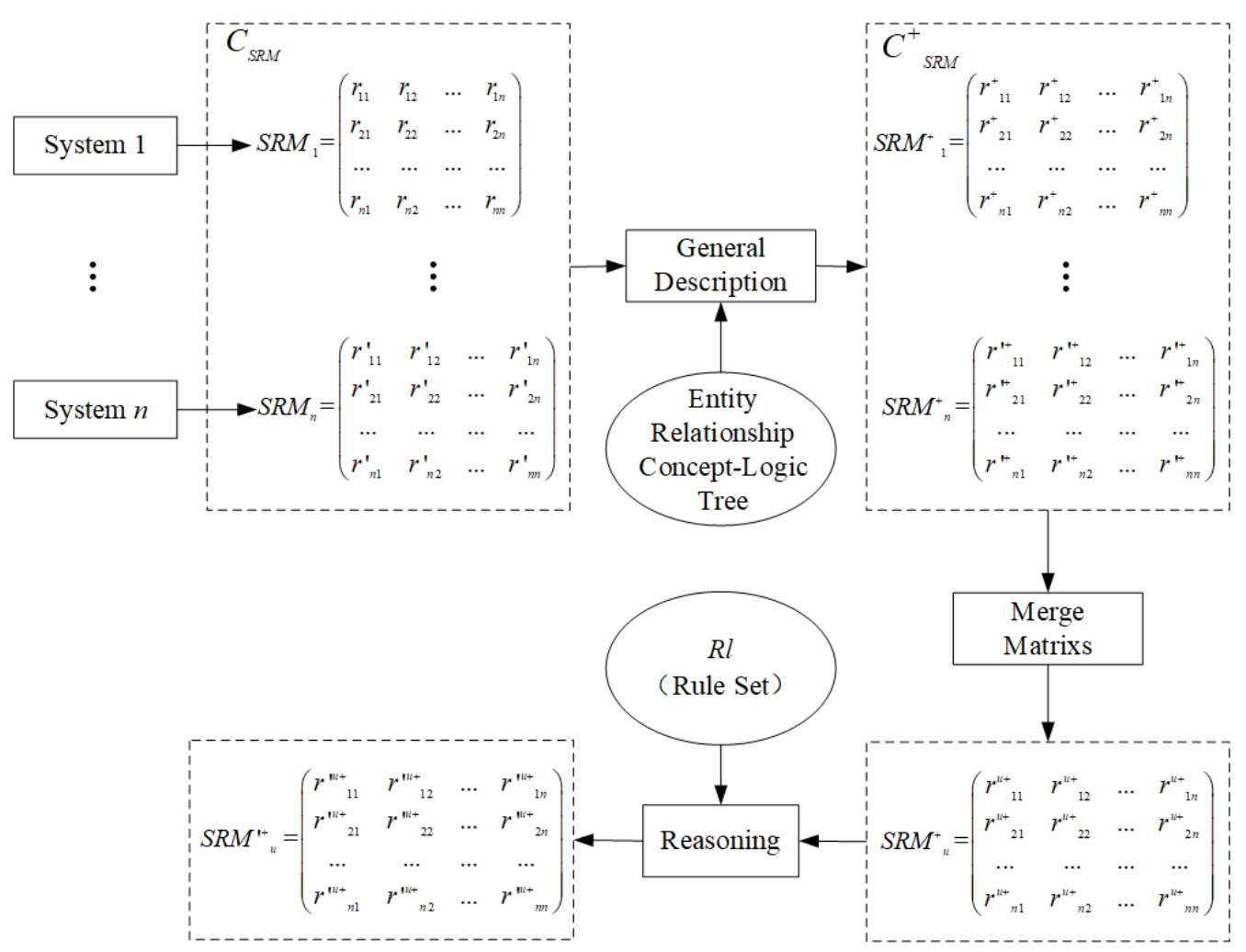

Figure 9. Structure of the entity class classification tree.

\section{Information Sharing Mechanism Based on Content}

Based on UBSIS, an implementation mechanism of information sharing was designed, which is shown in Figure 10.

Suppose both system $\mathbf{A}$ and system $\mathbf{B}$ contain the description information of entity $e_{i}$, and the description information in $\mathbf{A}$ or $\mathbf{B}$ merely reflect a certain aspect of it. Besides, the entity class set describing $e_{i}$ is $\left\{E C_{i}, E C_{i+1}, \ldots, E C_{i+n}\right\}(n \in \mathbf{N})$, in which $E C_{i}$ is a first level class. By organizing this information contained in the messages according to the information description model proposed above, bit oriented information can be transformed into content oriented structured information, so that the potential correlation between heterogeneous information can be expressed explicitly. After that, integrate the information describing the same entity on content and semantics. Then, update the UBSIS, and broadcast the newly received information to all systems except the one from which the information comes. In addition, when someone needs to get some information about specific entities, this can be done by a centralized query in UBSIS. Thus, information sharing among systems can be realized.

When considering the process of information sharing based on the UBSIS, it can be divided into two types: information broadcasting and information query. Among them, information broadcasting is easy to realize, e.g., when A finds something new and important, you just need to transport it to $\mathbf{B}$ through UBSIS. The process of querying and searching for the actual entity description information in UBSIS can be viewed as the process of searching for the required entity description information based on specific attributes and semantic relationships between entities; where the searching based on specific attributes is used to find the specific entities that meet the attribute requirements in the query conditions, while it can be easily realized based on the robust operational mechanism of XML; besides, the searching based on semantic relationships between entities is used to judge whether the entities found meet the semantic requirements in the query conditions; for example, when querying the information of entities with attribute $a_{i}=a_{1}$ and that possess relationship $r_{a}$ to $e_{i}$, while relationship $r_{b}$ to $e_{j}$. Firstly, entities with attribute $a_{i}=a_{1}$ are found through Algorithm 1, and then, we judge whether they possess $r_{a}$ to $e_{i}$ and relationship $r_{b}$ to $e_{j}$ through Algorithm 2. 


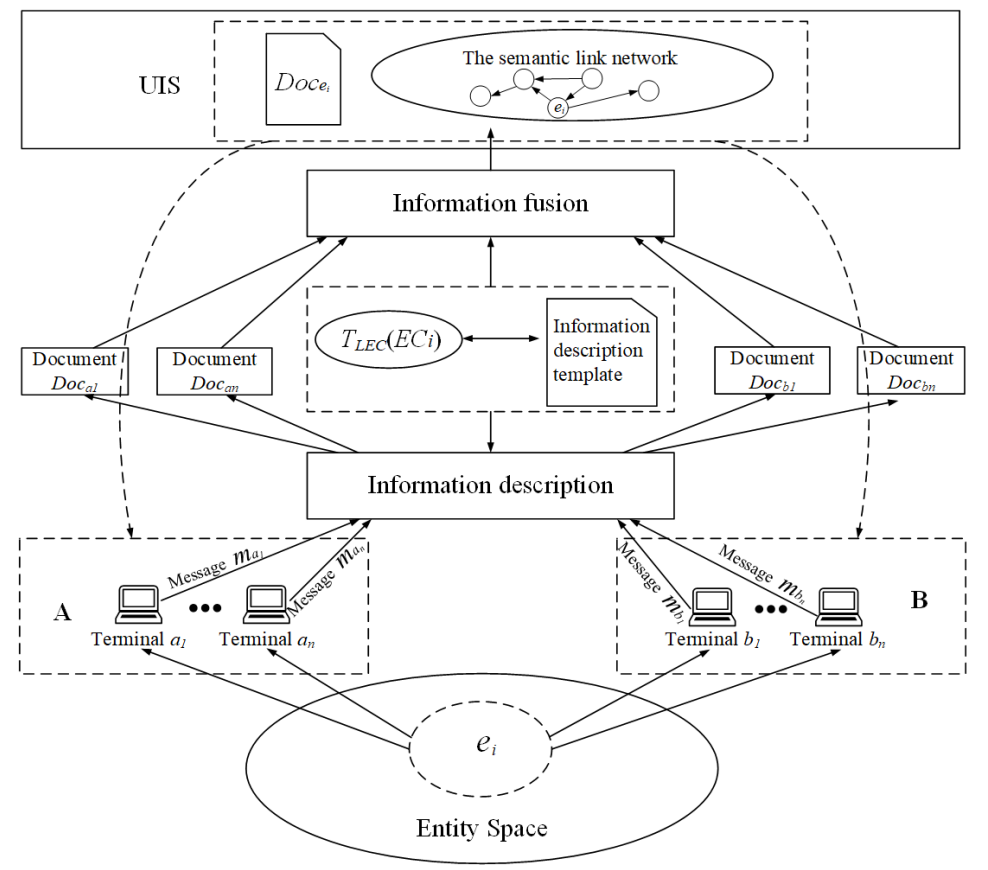

Figure 10. Implementation progress of information sharing.
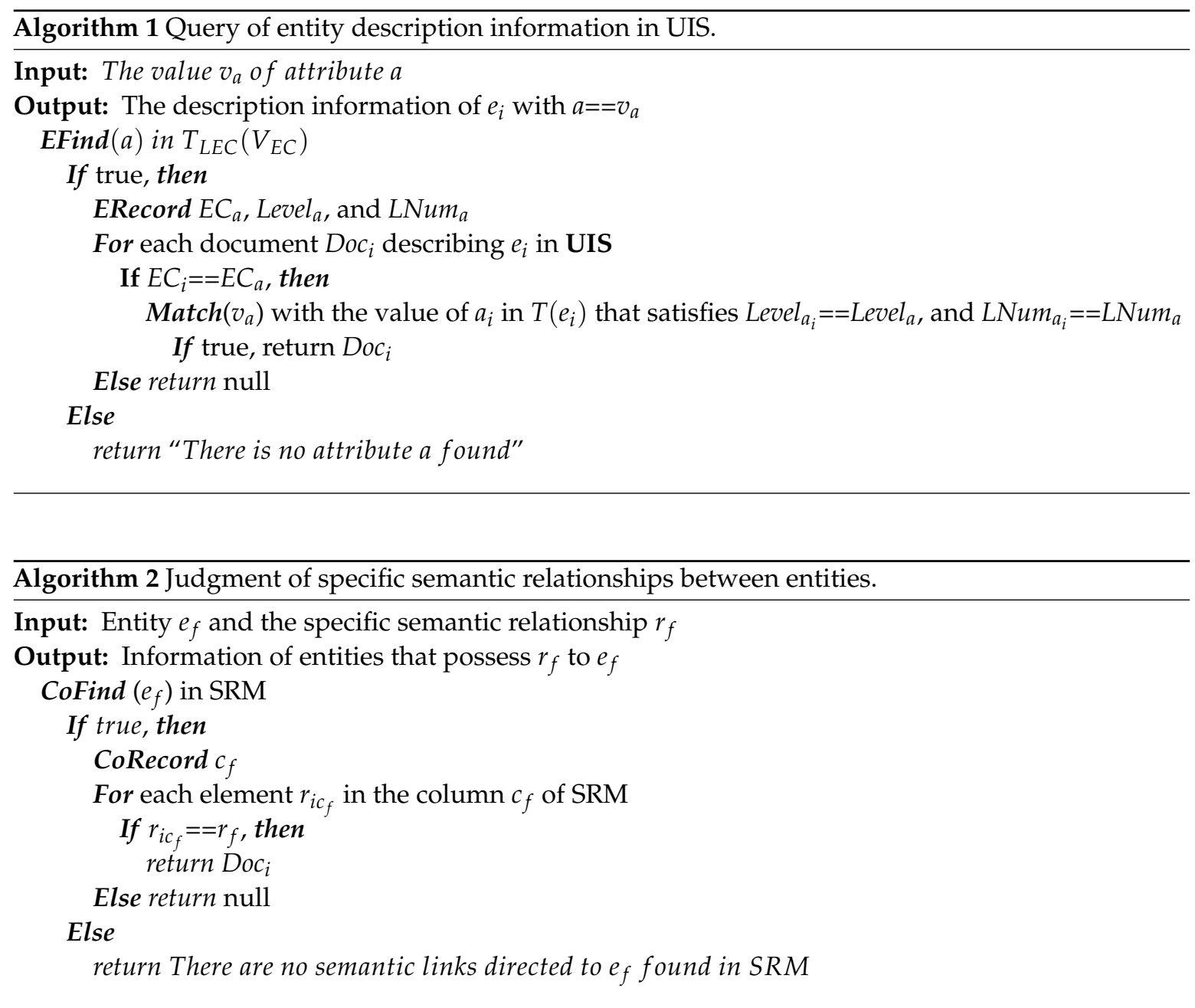
The Efind function is used to find whether there is an attribute $a$ in $T_{L E C}\left(V_{E C}\right)$. The ERecord function is used to record the position of the attribute $a$ in $T_{L E C}\left(V_{E C}\right)$, including the first level entity class $E C_{a}$ that it describes, as well as the level Level $l_{a}$ and the number $L N u m_{a}$ in Level $_{a}$ where it is located. $D o c_{i}$ represents the document stored in UIS that describes $e_{i}(i \in \mathbf{N})$, and the first level entity class that $e_{i}$ belongs to is $E C_{i}$. The Match function is used to match the value of $a_{i}$ in the particular position of $T\left(e_{i}\right)$ with $v_{a}$, to determine whether the entity $e_{i}$ is the one for which we are searching. The CoFind function is used to find the position of semantic links directed to $e_{f}$ in the first row of SRM. The CoRecord function is used to record the column number $c_{f}$ of the semantic links directed to $e_{f}$ in SRM. Doc $c_{i}$ represents the document stored in UIS that describes $e_{i}(i \in \mathbf{N})$, and the first level entity class that $e_{i}$ belongs to is $E C_{i}$.

Algorithm complexity analysis: Assume that there are $l$ nodes in $T_{L E C}\left(V_{E C}\right)$ and $n$ documents in the UBSIS.

- The time complexity: The average time complexity of finding attribute in $T_{L E C}\left(V_{E C}\right)$ is $\mathrm{O}(\log l)$, and the average time complexity of attribute matching in the UIS is $\mathrm{O}(n)$, then the average time complexity of this algorithm is $\mathrm{O}(n \log l)$. As for the semantic judging based on semantic links, SRM is used to find whether there are semantic links between entities, and the average time complexity is $\mathrm{O}(n)$. Thus, the average time complexity $T_{\text {avr }}$ of information searching based on specific attributes and semantic relationships between entities can be regarded as $\mathrm{O}(n(1+\log l))$.

- The space complexity: The average space complexity of finding attribute in $T_{L E C}\left(V_{E C}\right)$ is $\mathrm{O}(l)$, and the average space complexity of attribute matching in the UIS is $\mathrm{O}(n)$, then the average space complexity of this algorithm is $\mathrm{O}(n l)$. As for the semantic judging based on semantic links, the average space complexity is $\mathrm{O}\left(n^{2}\right)$. Thus, the average time complexity $S_{\text {avr }}$ of information searching based on specific attributes and semantic relationships between entities can be regarded as $\mathrm{O}(n(n+l))$.

In the traditional ontology based systems, graph matching and the subgraph indexing technique are usually used for information query. The total number of nodes in the graph is approximately $n(1+l / k)(k \in \mathbf{N} \wedge k \in[1, l])$, where $k$ is related to the number of entity classes, especially the number of first level entity classes. Both the average time complexity $T_{a v r}^{\prime}$ and the space complexity $S_{a v r}^{\prime}$ of finding required entities can be $\mathrm{O}\left(n d^{2}(1+l / k)\right)$ [26], where $d$ represents the average node-degree. Then, the ratio between $T_{a v r}$ and $T_{\text {avr }}^{\prime}$, which is denoted as TRto, is $(1+\log l) /\left(d^{2}(1+l / k)\right)$, while the ratio between $S_{\text {avr }}$ and $S_{\text {avr }}^{\prime}$, which is denoted as $S R t o$, is $(n+l) /\left(d^{2}(1+l / k)\right)$.

When $n$ increases, SRto will increases accordingly, which indicates that the space complexity of our method in information query is usually larger than that of graph matching. As for the time complexity, Figure 11 shows the relation of $k, l$, and $d$ when TRto $=1$, where we can see that:

- When $k$ is fixed, TRto decreases with the increase of $d$ or $l$, that is TRto will be smaller when the number of nodes in $T_{L E C}\left(V_{E C}\right)$, i.e., the number of categories, attributes, or states that describe entities is larger or the relationships among entities is more complex. For example, if $k=1$, i.e., all the entities belong to the same first entity class, then TRto is usually small than one as $d$ is usually larger than one.

- When $l$ is fixed, TRto decreases with the increase of $d$, i.e., TRto will also be smaller when the relationships among entities is more complex. Besides, TRto increases with the increase of $k$, i.e., TRto will be larger when the number of entity classes in $T_{L E C}\left(V_{E C}\right)$ is larger, which means that the less structural the categories, attributes, and states describing entities, the larger the TRto. 

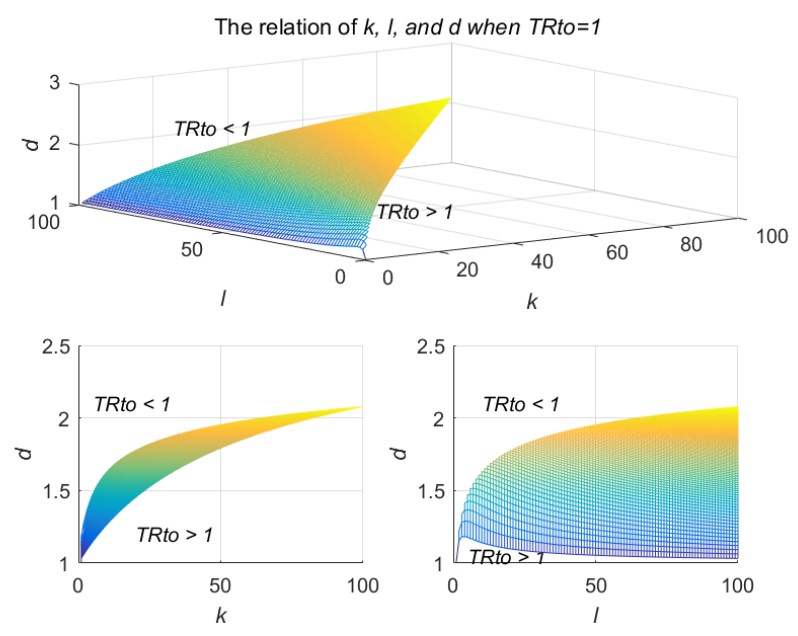

Figure 11. The relation of $k, l$, and $d$ when TRto $=1$.

Generally, since the concept-logic tree can be designed based on different topics and information systems usually only care about the information of targets relevant to their missions, the number of entities and entity classes can be controllable when describing a specific battlefield space, especially the number of first level entity classes. For example, when describing an entity on geographic coordinates, the concerned information mainly includes what it is, where it is, what it is doing, what it needs, and what state it is in. On the contrary, since entities are rarely completely isolated from the outside world, i.e., there exist semantic relationships between almost any two entities and the semantic relationships between every two entities may be more than one, which means that $d$ is usually large, if every two entities possesses a kind of relationship, then the value of $d$ can approximately be $(n-2) /(1+l / k)+$ 1 , which is much larger than two. The corresponding TRto will usually be smaller than one, and the larger the $d$, the smaller the TRto, which indicates that the time complexity of this content and semantic based method in information query is usually smaller than that of graph matching. To sum up, this content and semantic based algorithm adopts the idea of trading space for time, which will be useful in realizing sharing of battlefield situation information where the number of combat units is controllable and the requirement of real time is more important than space.

\section{Experiment Results and Analysis}

Based on above analysis and research, we took two combat information systems $\mathbf{A}$ and $\mathbf{B}$ as the specific research object to build the demonstration scene of heterogeneous information sharing, which is as shown in Figure 12, where the messages transmitted within $\mathbf{A}$ and $\mathbf{B}$ were in different formats; message simulator were used to generate formatted messages according to predefined message content or randomly; the sharing platform was used to realize information sharing between $\mathbf{A}$ and $\mathbf{B}$ based on the information sharing framework proposed in this paper; terminals were used to simulate the combat units working in $\mathbf{A}$ or $\mathbf{B}$.

When conducting the experiment, we took tanks as description targets and predefined the description information of 12 targets, which is shown in Figure 13c. The attribute concept-logic tree and the main entity relationships concerned are shown in Figure 13a,b respectively. In the process of the experiment, the complete information about Targets 1-4 and part of the information about 5-8 were transmitted within $\mathbf{A}$, while the complete information about Targets 9-12 and part of the information about $5-8$ were transmitted within $\mathbf{B}$, where the complete information about Targets 5-8 could be covered by the combination of the information transmitted within $\mathbf{A}$ and $\mathbf{B}$. The experimental content and result are as follows. 


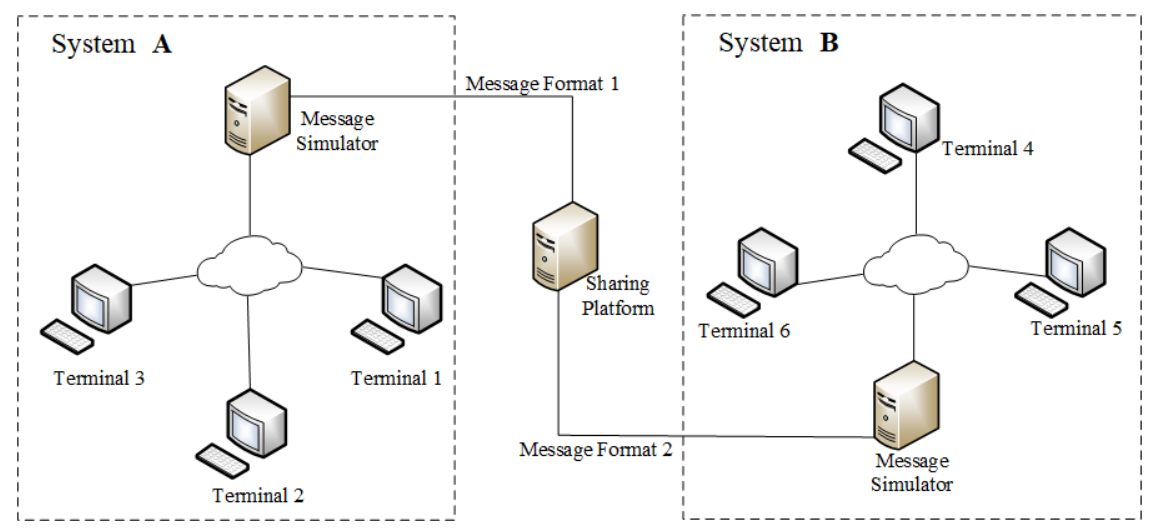

Figure 12. Demonstration scene of heterogeneous information sharing.

(1) Generate formatted messages according to the predefined description information of targets, where because the length of a single formatted message is limited, a piece of message usually contains a portion of the information about a target. Based on the information sharing platform, different terminals obtained different target information as required, and the information requirements are shown in Table 4.

When the system was stable, we compared the information obtained by different terminals with the predefined description information of targets, where a matching degree between obtained information and predefined information, which is denoted as $M d e_{u}$, was used to measure the validity and correctness of information acquisition.

The value of $M d e_{u}$ can be calculated according to Equation (8), where $N_{j}$ represents the total number of items describing the information about target $j, \mathrm{OI}\left(\mathrm{Item}_{i}\right)$ represents the value of the $i$ th item in the obtained information about target $j, \operatorname{PI}\left(\mathrm{Item}_{i}\right)$ represents the value of the $i$ th item in the predefined information about target $j$, and $M$ represents the number of targets in the information requirement of a terminal. If $\mathrm{OI}\left(\mathrm{Item}_{i}\right)$ matches $\mathrm{PI}\left(\mathrm{Item}_{i}\right)$, then the value of $\mathrm{OI}\left(\mathrm{Item}_{i}\right) / \mathrm{PI}\left(\mathrm{Item}_{i}\right)$ is one, otherwise the value of $\mathrm{OI}\left(\mathrm{Item}_{i}\right) / \mathrm{PI}\left(\mathrm{Item}_{i}\right)$ is zero.

$$
\left\{\begin{array}{c}
M d e_{j}=\sum_{i}^{N_{j}} \frac{\mathrm{OI}\left(\mathrm{Item}_{i}\right) / \mathrm{PI}\left(\mathrm{Item}_{i}\right)}{N_{j}} \\
M d e_{u}=\sum_{j}^{M} M d e_{j} / M
\end{array}\right.
$$

(2) On the basis of Step 1, modify some of the predefined information about some targets randomly, while other conditions and experimental steps remain unchanged. Calculate the information matching degree of each terminal.

(3) Repeat Step 2 several times, while calculating the information matching degree of each terminal.

Table 4. Information requirements of different terminals.

\begin{tabular}{cl}
\hline Terminal & Information Requirement \\
\hline 1 & Information about Targets $1,2,5$, and 6. \\
2 & Information about Targets $3,4,7$, and 8. \\
3 & Information about Targets $1,6,9$, and 12. \\
4 & Information about Targets $2,7,10$, and 11. \\
5 & Information about targets commanded by Target 2. \\
6 & Information about targets cooperated with Target 9. \\
\hline
\end{tabular}




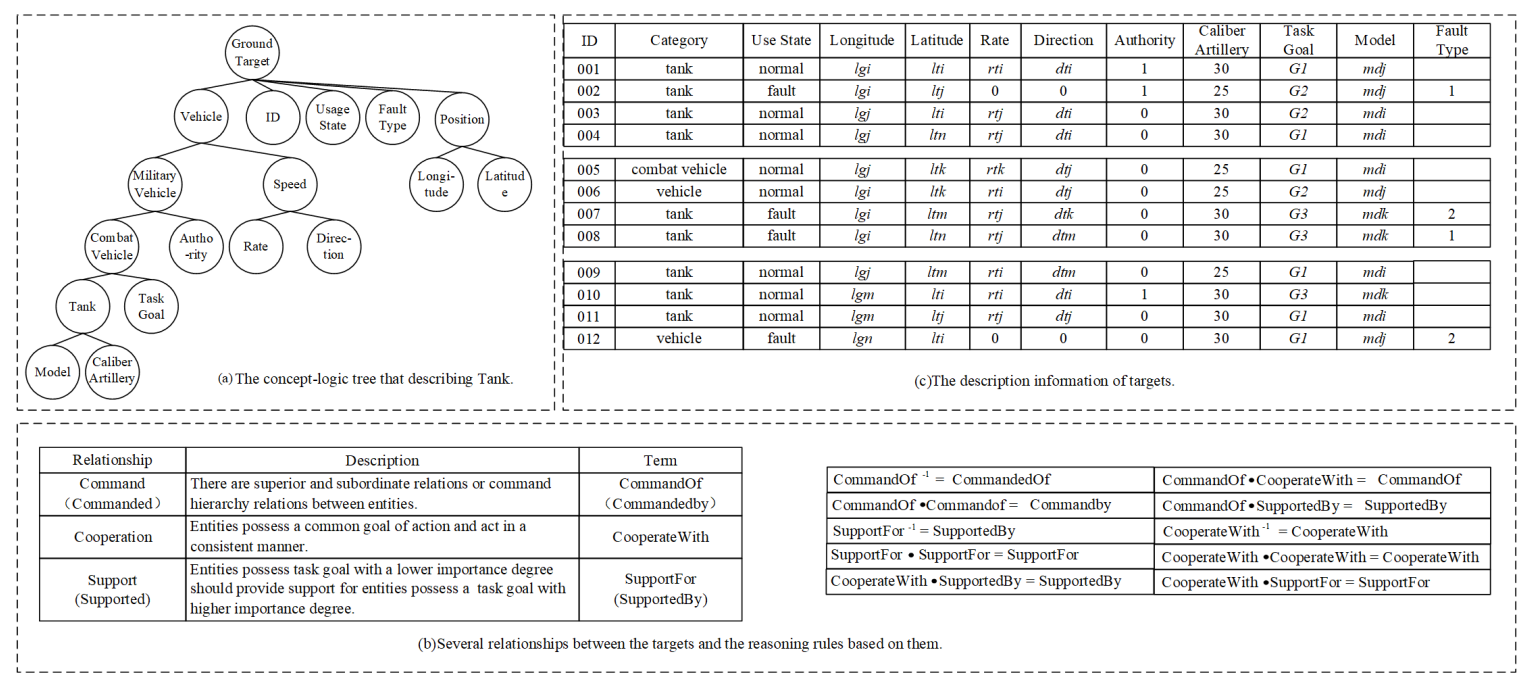

Figure 13. Information about targets, the concept-logic tree, and entity relationships used during the experiment.

The results of the total experiment is showed in Figure 14.

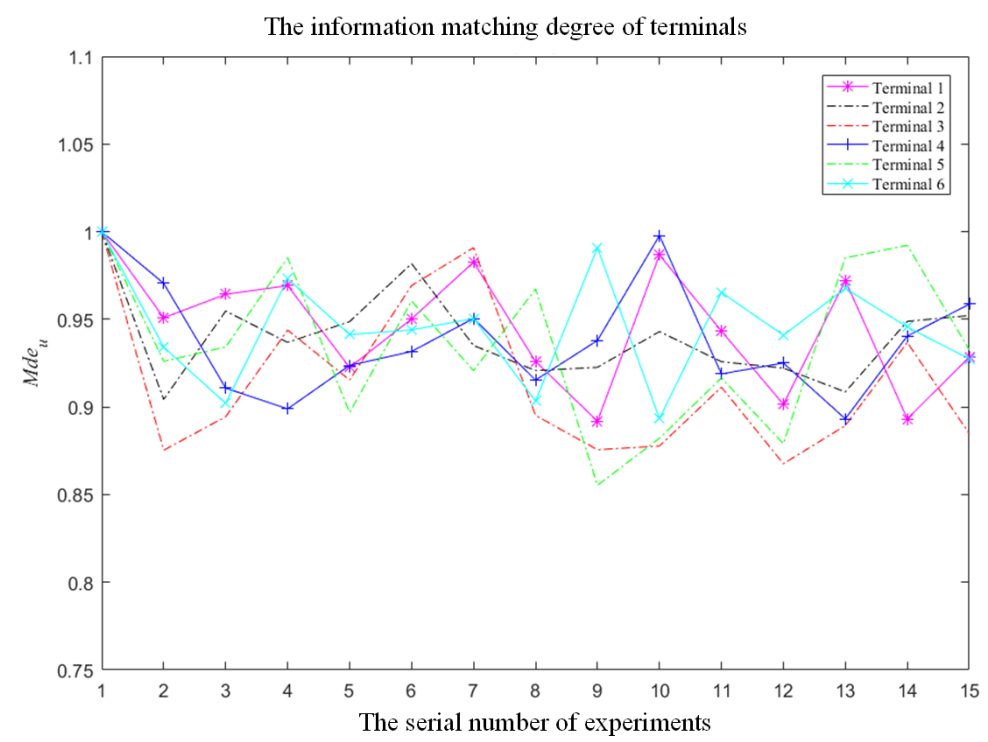

Figure 14. The information matching degree of terminals.

Before the predefined information about targets was modified, the information matching degree of each terminal was one, i.e., each terminal could obtain the required information completely and correctly based on the information sharing platform. After the predefined information about targets was modified randomly, the information matching degree of each terminal showed some fluctuations. That is, some items of the obtained information were mismatched with the predefined information. The main reason was that the semantic integrity of the information contained in the message was not fully considered when updating the unified information space. For example, there was an incidence relation between $a_{1}$ and $a_{2}$, and the update of their information should maintain the consistency of time. However, due to the limited length of the message, a message may only contain the information of $a_{1}$ or $a_{2}$. If updating the unified information space with the content of this message, then a mismatch in the information of $a_{1}$ and $a_{2}$ occurred with respect to the time. That is, the information of $a_{1}$ or $a_{2}$ was not the latest information, which correspondingly caused the mismatch of the obtained information and the predefined information when computing the information matching degree of terminals. Thus, the verification of the information integrity needs to be further improved. 


\section{Conclusions}

In this paper, we aimed at realizing efficient information sharing among different information systems in a heterogeneous battlefield situation. We designed a heterogeneous information sharing framework based on a unified battlefield situation information space and realized the entity oriented information sharing based on the content. The theoretical analysis and experimental results showed that effective information sharing and interaction between different systems could be realized according to the information sharing platform with the framework we proposed. The information query efficiency was faster than graph matching, which is usually used in ontology based systems. In the future work, the approach of realizing the verification of information integrity and solving information conflicts in the aspect of semantics will be studied to expand the efficiency and correctness of information sharing.

Author Contributions: Conceptualization, C.L. and W.Z.; methodology, C.L. and W.Z.; software, W.Z.; validation, W.Z., A.L., and J.Z.; writing, original draft preparation, C.L.; writing, review and editing, W.Z., A.L., and J.Z. All authors read and agreed to the published version of the manuscript.

Funding: This research received no external funding.

Conflicts of Interest: The authors declare no conflict of interest.

\section{Abbreviations}

The following abbreviations are used in this manuscript:

FDBS Federated database system

DWH Data warehouse

BSIS Battlefield situation information space

UBSIS Unified battlefield situation information space

\section{References}

1. Li, H.; Tang, X.; Yang, Y. Information integration and service optimization in marine battlefield. In Proceedings of the 2008 International Conference on Information and Automation, Zhangjiajie, China, 20-23 June 2008; pp. 1703-1707. [CrossRef]

2. Kim, A.H.; Macha, C. Providing the Tools for Information Sharing: Net-Centric Enterprise Services. CrossTalk J. Def. Softw. Eng. 2007, 20, 16-18.

3. Gupta, A.M.; Gadepally, V.; Stonebraker, M. Cross-engine query execution in federated database systems. In Proceedings of the 2016 IEEE High Performance Extreme Computing Conference (HPEC), Waltham, MA, USA, 13-15 September 2016; pp. 1-6. [CrossRef]

4. Bater, J.; Elliott, G.; Eggen, C.; Goel, S.; Kho, A.; Rogers, J. SMCQL: Secure Querying for Federated Databases. Proc. VLDB Endow. 2017, 10, 673-684. [CrossRef]

5. Mohamme, M.A.; Hasso, A.R.; Shawka, A.R.; Al-Khafaj, N.J. E-government architecture uses data warehouse techniques to increase information sharing in Iraqi universities. In Proceedings of the 2012 IEEE Symposium on E-Learning, E-Management and E-Services, Kuala Lumpur, Malaysia, 21-24 October 2012; pp. 1-5.

6. Lijing, Z.; Da, W.; Weihua, P. The XML based Information Sharing Technology for the Power Enterprise. In Proceedings of the 2009 International Forum on Information Technology and Applications, Chengdu, China, 15-17 May 2009; pp. 11-13. [CrossRef]

7. Bellahsene, Z., Bonifati, A., Rahm, E. Schema Matching and Mapping. Data-Centric Systems and Applications; Springer: Berlin/Heidelberg, Germany, 2011; Volume 30, pp. 121-160.

8. Liu, C.; Zhao, W.; Tian, C. A Method of Battlefield Information Fusion Based on Structured Knowledge Concept Tree. In Proceedings of the 2018 14th International Conference on Semantics, Knowledge and Grids (SKG), Guangzhou, China, 12-14 September 2018; pp. 167-172. [CrossRef]

9. Qiao, S; Liu, J. Method of Battlefield Target Information Sharing Based on Ontology. In Proceedings of the 2017 4th International Conference on Information Science and Control Engineering, Changsha, China, 21-23 July 2017.

10. Song, J.F.; Zhang, W.M.; Xiao, W.D.; Tang, D.Q. Study on battlespace ontology construction approach. Int. J. Intell. Syst. 2005, 20, 1219-1231. [CrossRef] 
11. Shan, W.F.; Zhou, J.W.; Dai, H.J.; Wang, P. A New Method of Battlefield Situation Collaborative Plotting Based on Ontology. App. Mech. Mater. 2014, 513-517, 1365-1371. [CrossRef]

12. Shan, W.; Zhou, J.; Hu, H.; Wang, P. Battlefield Situational Information Discovery Based on Ontology. In Proceedings of the 2013 International Conference on Computer Sciences and Applications, Wuhan, China, 14-15 December 2013; pp. 719-722.

13. Zhuge, H.; Zhang, J. Automatically constructing semantic link network on documents. Concurr. Comput. Pract. Exp. 2011, 23, 956-971. [CrossRef]

14. Zhuge, H. Inheritance rules for flexible model retrieval. Decis. Support Syst. 1998, 22, 379-390. [CrossRef]

15. Zhuge, H. Active e-document framework ADF: Model and tool. Inf. Manag. 2003, 41, 87-97. [CrossRef]

16. Zhuge, H. Socio-natural thought semantic link network: A method of semantic networking in the Cyber Physical Society. In Proceedings of the 24th IEEE International Conference on Advanced Information Networking and Applications (AINA), Perth, WA, Australia, 20-23 April 2010.

17. Zhuge, H.; Jia, R.; Liu, J. Semantic link network builder and intelligent semantic browser. Concurr. Comput. Pract. Exp. 2004, 16, 1453-1476. [CrossRef]

18. Zhuge, H. The Semantic Link Network. In The Knowledge Grid: Toward Cyber-Physical Society; World Scientific: Singapore, 2012.

19. Zhuge, H.; Xu, B. Basic operations, completeness and dynamicity of cyber physical socio semantic link network CPSocio-SLN. Concurr. Comput. Pract. Exp. 2011, 23, 924-939. [CrossRef]

20. Zhuge, H. Semantic linking through spaces for cyber-physical-socio intelligence: A methodology. Artif. Intell. 2011, 175, 988-1019. [CrossRef]

21. Zhuge, H. The emerging structures. In Multi-Dimensional Summarization in Cyber-Physical Society; Morgan Kaufmann: Burlington, MA, USA, 2016.

22. Sun, X.; Zhuge, H. Summarization of Scientific Paper through Reinforcement Ranking on Semantic Link Network. IEEE Access 2018, 6, 40611-40625. [CrossRef]

23. Zhuge, H. Cyber-Physical-Social Intelligence on Human-Machine-Nature Symbiosis, Springer: Berlin/Heidelberg, Germany, 2019.

24. Franz, T.; Schultz, A.; Sizov, S.; Staab, S. TripleRank: Ranking Semantic Web Data by Tensor Decomposition. In International Semantic Web Conference, Proceedings of the International Semantic Web Conference Semantic-ISWC 2009, Chantilly, VA, USA, 25-29 October 2009; Lecture Notes in Computer Science; Bernstein, A., Karger, D.R., Heath, T., Feigenbaum, L., Maynard, D., Motta, E., Thirunarayan, K., Eds.; Springer: Berlin/Heidelberg, Germany, 2009; Volume 5823, pp. 213-228.

25. Zhuge, H.; Sun, Y.; Zhang, J. Schema Theory for Semantic Link Network. In Proceedings of the 2008 Fourth International Conference on Semantics, Knowledge and Grid, Beijing, China, 3-5 December 2008; pp. 189-196.

26. Khan, A.; Li, N.; Yan, X.; Guan, Z.; Chakraborty, S.; Tao, S. Neighborhood based fast graph search in large networks. In Proceedings of the 2011 ACM SIGMOD International Conference on Management of Data, Athens, Greece, 12-16 June 2011; pp. 901-912

(c) 2019 by the authors. Licensee MDPI, Basel, Switzerland. This article is an open access article distributed under the terms and conditions of the Creative Commons Attribution (CC BY) license (http://creativecommons.org/licenses/by/4.0/). 